\title{
Honeycomb phononic crystals with self-similar hierarchy
}

\author{
Davood Mousanezhad, ${ }^{1}$ Sahab Babaee, ${ }^{2}$ Ranajay Ghosh, ${ }^{1}$ Elsadig Mahdi, ${ }^{3}$ Katia Bertoldi, ${ }^{2}$ and Ashkan Vaziri ${ }^{1, *}$ \\ ${ }^{1}$ Department of Mechanical and Industrial Engineering, Northeastern University, Boston, Massachusetts 02115, USA \\ ${ }^{2}$ Harvard John A. Paulson School of Engineering and Applied Sciences, Harvard University, Cambridge, Massachusetts 02138, USA \\ ${ }^{3}$ Mechanical and Industrial Engineering Department, Qatar University, Doha, Qatar
}

(Received 1 May 2015; published 29 September 2015)

\begin{abstract}
We highlight the effect of structural hierarchy and deformation on band structure and wave-propagation behavior of two-dimensional phononic crystals. Our results show that the topological hierarchical architecture and instability-induced pattern transformations of the structure under compression can be effectively used to tune the band gaps and directionality of phononic crystals. The work provides insights into the role of structural organization and hierarchy in regulating the dynamic behavior of phononic crystals, and opportunities for developing tunable phononic devices.
\end{abstract}

DOI: 10.1103/PhysRevB.92.104304

PACS number(s): 63.20.D-, 43.35.+d, 62.30.+d

Hierarchical organization is ubiquitous in biological systems, from the nanometer to the macroscopic length scales. Examples include collagen [1], bone [2,3], tooth [2], tendon [3], wood [3,4], nacre [5], gecko foot pads [6], Asteriscus plant [7], Euplectella sponge [8], and water-repellent biological systems [9]. The purely structural role of hierarchy in boosting mechanical performance is now well known [10-14]. In addition to hierarchy, periodic organizations aimed at influencing the wave-propagation behavior, for instance in structural colorations, can also be found in nature [15-17]. More interestingly, the reversible modulation of these so-called photonic crystals through deformation provides an incredibly rich optical behavior enhancing their survival [18,19].

Pursuing these synergetic motifs for materials development [20-23], we investigate a different class of hierarchical organization based on two-dimensional (2D) honeycomblike structures primarily geared towards phononic applications (i.e., phononic crystals) and the effect of deformation on controlling their band gaps (defined as frequency ranges of strong wave attenuation). To this end, 2D lattices with different nonhierarchical topologies have been well investigated (no deformation included) [24-26]. However, recently, Xu et al. [27] investigated wave propagation in 2D hexagonal lattice structures with sandwich plate cell walls possessing only firstorder non-self-similar hierarchy. This early study, although lacking a systematic analysis of the role of hierarchy in fostering phononic properties, provided an early evidence of expansion of band gaps with hierarchy.

In this paper, we study the effect of structural hierarchy on the band structure and directionality of these crystals, as well as investigate the additional effect of compressive loads on the tunability of band gaps of first-order hierarchical phononic crystals. Interestingly, our results reveal that hierarchy and pattern transformations through compression can significantly affect the dynamic response, and they can be effectively used to tune the propagation of elastic waves in phononic crystals.

The evolution of a regular honeycomb into the fractal-like hierarchical phononic crystal studied in this paper is illustrated schematically in Fig. 1. The structural organization of the

\footnotetext{
*vaziri@coe.neu.edu
}

honeycomb at each order of hierarchy $\left(\gamma_{i}\right)$ is defined by the ratio of the newly introduced hexagonal edge length $\left(l_{i}\right)$ to the previous hexagon's edge length $\left(l_{i-1}\right)$, i.e., $\gamma_{i}=l_{i} / l_{i-1}$. For convenience, $\gamma_{1}$ is defined as $\gamma_{1}=2 l_{1} / l_{0}$ [see Fig. 1(b)] [10]. The following geometrical constraints must be imposed on the structure to avoid overlapping edges:

$$
\left\{\begin{array}{l}
0 \leqslant \gamma_{n} \leqslant 1 \\
\sum_{i=1}^{n} \prod_{j=1}^{i} \gamma_{j} \leqslant 1,
\end{array}\right.
$$

where $n$ is the order of hierarchy $(n \geqslant 1)$. The dimensionless relative density (equal to area fraction) of the structure [compared to the material density $\left(\rho_{s}\right)$ ], i.e., $\rho_{c}=\rho / \rho_{s}$, is given as the following:

$$
\rho_{c}=\frac{2}{\sqrt{3}}\left(1+\sum_{i=1}^{n} 3^{i-1} \prod_{j=1}^{i} \gamma_{j}\right) \frac{t_{n}}{l_{0}},
$$

where $t_{n}$ is the wall thickness, which for simplicity is assumed to be uniform throughout the structure. Thus, thickness $\left(t_{n}\right)$ must decrease to maintain a fixed relative density $\left(\rho_{c}\right)$ as the order of hierarchy $(n)$ and the values of $\gamma_{i}$ are increased.

We consider the structure to be infinitely extended in 2D space and the advancing wave front to induce no finite strains to model waves of low intensity with wavelengths of the order of lattice characteristic size. Numerical simulations of the propagation of these small-amplitude elastic waves in the crystal (in undeformed configuration) were performed using the finite-element (FE) method and Bloch wave analysis $[28,29]$. In the FE models, honeycomb walls were modeled as Timoshenko beams [30] with a rectangular cross section of unit length normal to the plane of wave motion, and the material was assumed to be aluminum with Young's modulus $E_{s}=71 \mathrm{GPa}$, Poisson's ratio $v_{s}=0.33$, and density $\rho_{s}=2700 \mathrm{~kg} / \mathrm{m}^{3}$. The relative density of the structure was kept constant at $8 \%$. The frequency of the propagating wave $(\omega)$ was normalized with respect to the first flexural frequency of a simply supported beam with length $l_{0}$ and thickness $t_{0}$, that is, $\Omega=\omega / \omega_{0}$, where $\omega_{0}=\pi^{2} \sqrt{E_{s} t_{0}{ }^{2} /\left(12 \rho_{s} l_{0}{ }^{4}\right)}$ [25].

We compare, in Fig. 2, the band structures and directionality diagrams of a regular honeycomb (left column) and a firstorder hierarchical structure with $\gamma_{1}=0.5$ (right column). In 
(a) Regular honeycomb

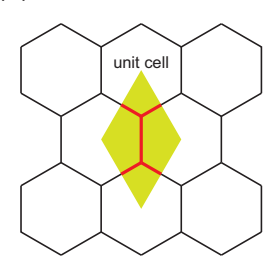
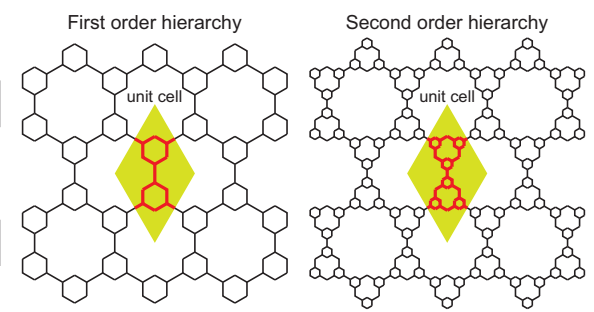

(b)

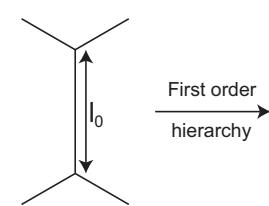

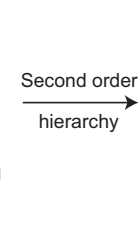

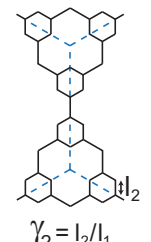

FIG. 1. (Color online) Hierarchical honeycombs. (a) The evolution of a regular hexagonal honeycomb (left) to first-order (middle) and second-order (right) hierarchical honeycombs. (b) Corresponding primitive unit cells of the structures.

Fig. 2(a), we report the normalized frequency as a function of the reduced wave vector [29]. For the considered frequency range, the regular structure features a band gap at $\Omega=$ 4.56-5.46. On introducing hierarchy, the lowest frequency band gap is now much lower $(\Omega=2.01-2.85)$ and several other new band gaps appear in the considered frequency range $(\Omega=5.77-10.22,11.67-12.38)$. Another preexisting band gap is also shifted to the lower frequency at $\Omega=$ 13.16-13.55. This considerable change in the band structure is due to the added hexagons (brought about by hierarchy), which also reduce the cell wall thickness to conserve mass. This geometrical change results in an increase in multiple scattering of the propagating waves at the cell walls and consequently opening up the Bragg-type band gaps [31]. The alterations of the band structure indicate a hierarchy-dependent transition, which parallels the effect of hierarchy on mechanical behavior in other contexts [10-14].

Since band diagrams such as Fig. 2(a) cannot always fully provide the directional behavior of a lattice structure, they are often investigated using dispersion surfaces (in compact form: phase and group velocities) [32]. In rapidly expanding broadband applications, it is crucial to focus on the low-frequency regime in addition to more well-investigated high-frequency behavior [24,33,34]. Figures 2(b) and 2(c), respectively, present the phase and group velocity profiles for the lowest two modes of propagating waves at relatively low frequency of $\Omega=0.1$. Each velocity profile is normalized with respect to the maximum velocity of the profile $\left(V_{\max }\right)$ [see Fig. 2(b)]. For the regular structure (left column), the phase velocity profile exhibits a slight preference in the direction of propagation at $\theta=0^{\circ}, \theta=60^{\circ}$, and due to symmetry, at $\theta=$ $120^{\circ}$ for mode 1 (shear or S mode), whereas it does not show any preferential direction for mode 2 (longitudinal or L mode). Hierarchy seems to have no effect on the relative isotropy of mode 2. However, anisotropy of mode 1 is further accentuated due to hierarchy. The origin of this effect can be traced to decreased thickness of the original cell walls due to additional hexagons resulting in an increase in the relative anisotropy ratio $V_{\max } / V_{\min }$. Group velocity, which typically indicates

(a)
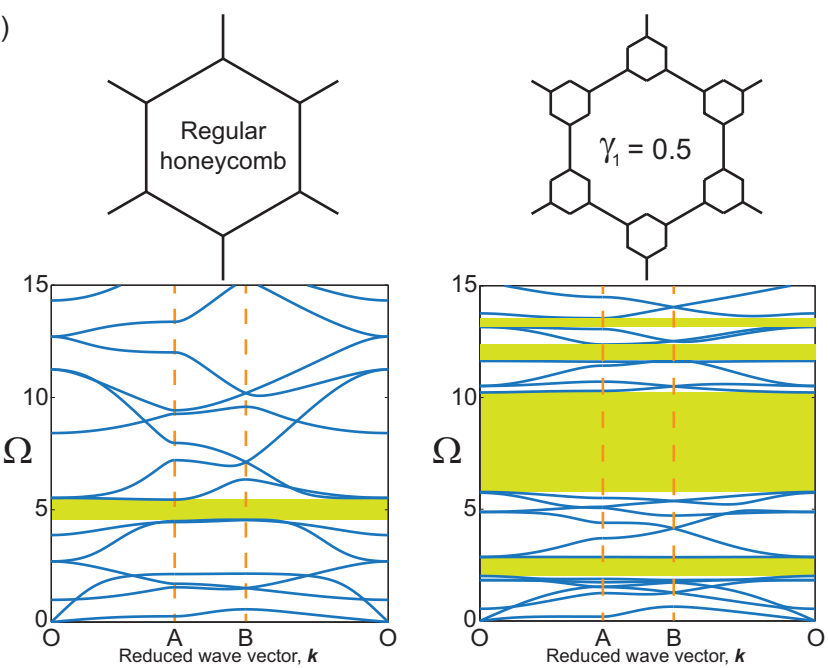

(b)
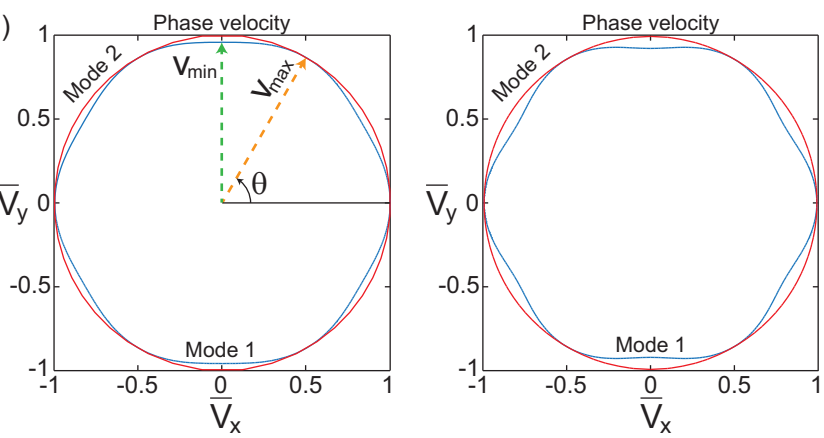

(c)
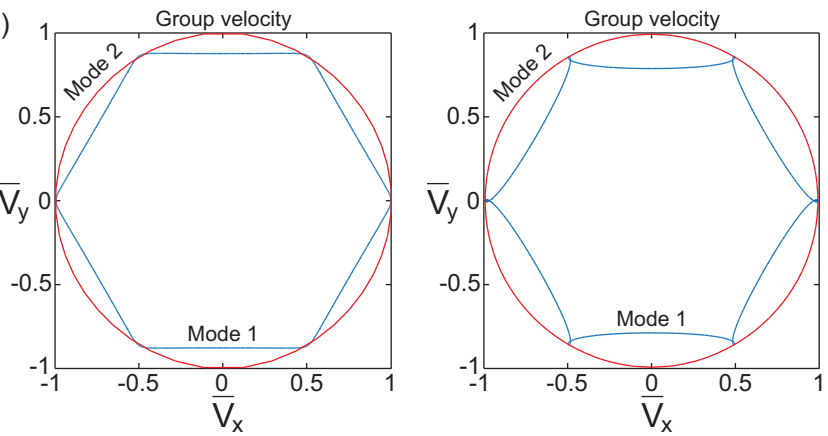

FIG. 2. (Color online) Regular hexagonal honeycomb vs a firstorder hierarchical honeycomb with $\gamma_{1}=0.5$. (a) Effect of hierarchy on band gaps. (b), (c) Effects of hierarchy on the directionality of phase and group velocities.

the velocity at which energy is transported along the wave vector, also reflects this anisotropy concentration of mode 1 in Fig. 2(c) for both the regular and hierarchical structures.

Next, we investigate the effects of the hierarchical order (n) and geometrical ratios $\left(\gamma_{i}^{\prime} s\right)$ on band gaps and wave directionality. For convenience, we limited the analysis to selfsimilar hierarchical honeycombs up to third order of hierarchy with self-similarity ratio, $\eta$, defined as $\eta=\gamma_{n}=\gamma_{n-1}=\cdots=$ $\gamma_{1}$ for $n$th order of hierarchy with $n=1,2$, and 3 , respectively, for first, second, and third orders of hierarchy. The geometrical constraints given in Eq. (1) limit the maximum value of $\eta$ at each order of hierarchy to $\eta_{\max }=1,0.62$, and 0.54 , respectively for first, second, and third orders of hierarchy.

Figures 3(a)-3(c) show the evolution of band gaps as the value of $\eta$ increases, respectively, for first, second, and third 
(a)

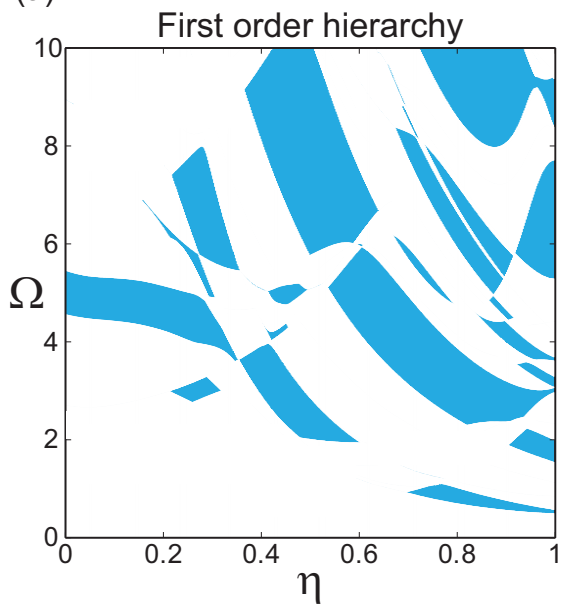

(b)

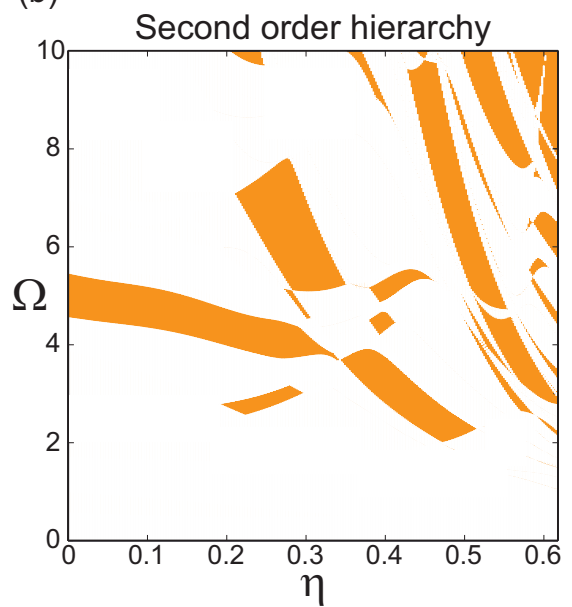

(c)

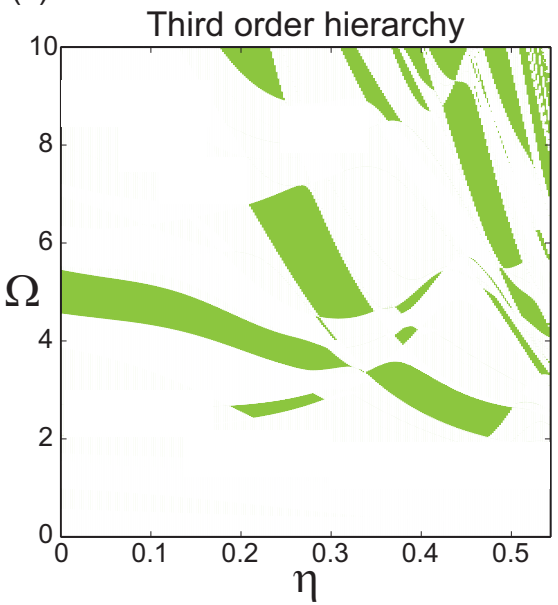

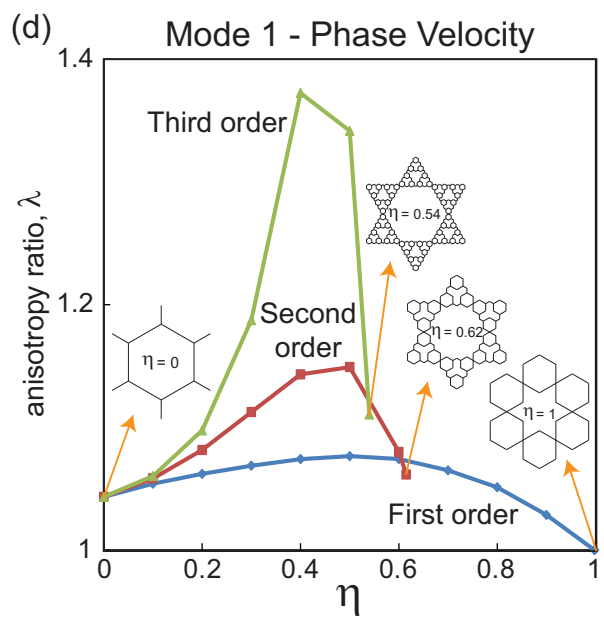

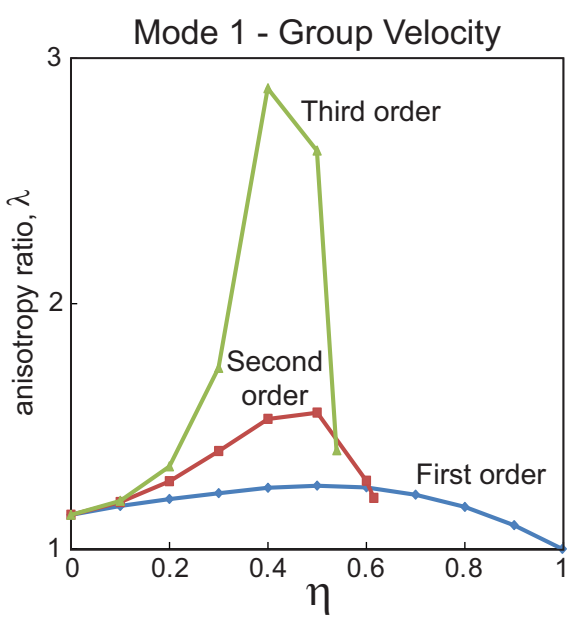

FIG. 3. (Color online) (a)-(c) The evolution of band gaps as a function of self-similarity ratio ( $\eta$ ) for hierarchical honeycombs with first, second, and third orders of hierarchy. (d) The evolution of anisotropy ratio $(\lambda)$ as a function of self-similarity ratio $(\eta)$ for phase, and group velocity profiles for mode 1 of propagating waves.

orders of hierarchy for the range of frequencies $0 \leqslant \Omega \leqslant 10$. Note that the upper bound of the horizontal axis is limited to $\eta_{\max }$. For $n=1$ [Fig. 3(a)], introducing hierarchy is found to, at first, lower the midgap position of the original nonhierarchical band gap, although the width of the band gap remains fairly the same. Thereafter, a slender band gap appears on either side of this central band gap. Increasing $\eta$ opens up another band gap at higher frequencies, which continues to expand as the original dominant band narrows. After $\eta \approx 0.35$, this original band gap disappears, giving way to two separate band-gap flanges which first expand and then disappear, giving further band-gap flanges. This waxing and waning pattern of band gaps continues as newer band gaps emerge and disappear. Similar phenomena are observed for higher orders of hierarchy [see Figs. 3(b) and 3(c)]. The results presented in Figs. 3(a)-3(c) confirm that the order of hierarchy and self-similarity ratio are two important geometric parameters affecting the band structure. This has important implications on the critical gap/midgap ratio for phononic applications [35].

To study the directionality of propagating waves at low frequency of $\Omega=0.1$, we define the following scalar anisotropy ratio [31]:

$$
\lambda=V_{\max } / V_{\min },
$$

where $V_{\max }$ and $V_{\min }$ are the maximum and minimum wave velocities, respectively [Fig. 2(b)]. Note that $\lambda \geqslant 1$, with $\lambda=1$ showing an isotropic media where elastic waves propagate with the same speed in every spatial direction with no preference (i.e., the polar plot of wave velocity is circular). We report in Fig. 3(d) the evolution of anisotropy ratio as $\eta$ increases for phase and group velocity profiles for mode 1 of propagating waves. Note that the phase and group velocity profiles of the structures for mode 2 do not show any preferential direction of propagation indicating isotropic response (i.e., $\lambda=1$ ). Figure 3(d) shows that for mode $1, \lambda$ of both the phase and group velocity profiles rise from the initial values $(\eta=0$, regular structure) up to a turning point (critical point), then decrease as a function of $\eta$, restoring isotropy. This behavior is entirely due to the redistribution of matter within the crystal resulting in an initial increase of scattering in the smaller hexagons, whose expanding size eventually restores crystal symmetry. The figures also reveal that the higher order of hierarchy accentuates the rate of anisotropy ratio. This is due to higher 
(a)

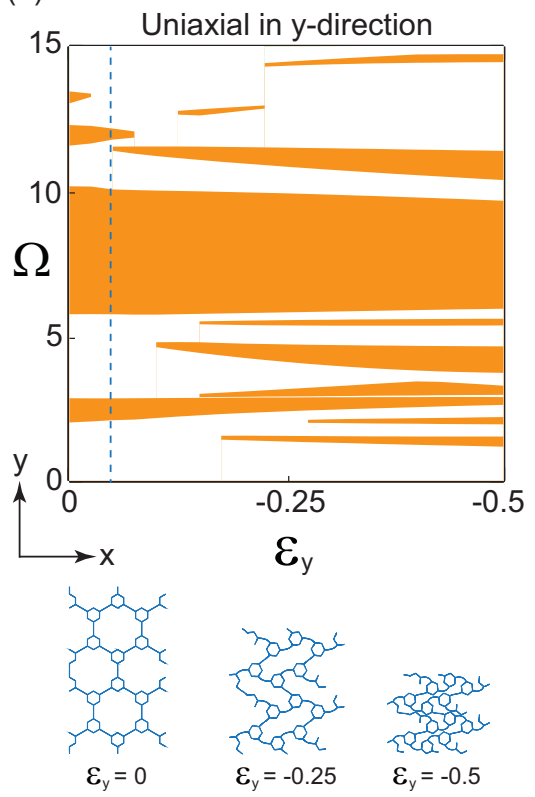

(b)
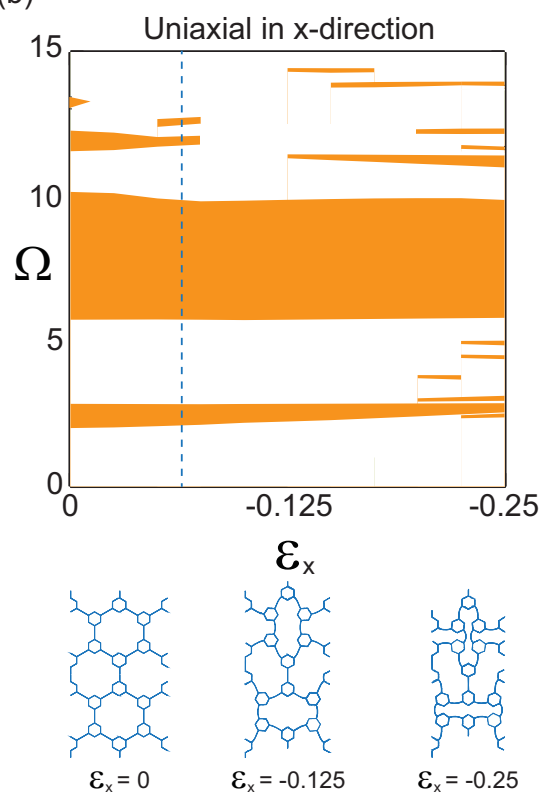

$\varepsilon_{\mathrm{x}}$

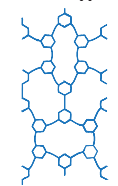

$\varepsilon_{\mathrm{x}}=-0.125$

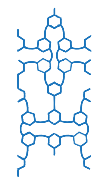

$\varepsilon_{\mathrm{x}}=-0.25$ (c)

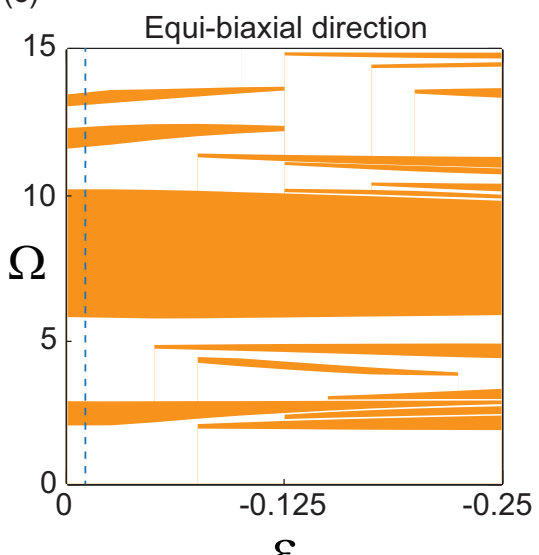

$\varepsilon$

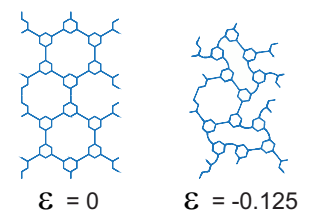

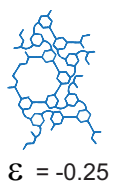

FIG. 4. (Color online) The evolution of band gaps as a function of the applied engineering strain for the first-order hierarchical honeycomb with $\gamma_{1}=0.5$ subjected to (a) uniaxial compression in the $y$ direction, (b) uniaxial compression in the $x$ direction, and (c) equibiaxial compression. The dashed vertical lines represent the strain such that buckling occurs. The undeformed and deformed configurations of the RVEs at different levels of applied strains are shown at the bottom.

number of smaller hexagons in the structure, which introduces greater incremental anisotropy in the crystal structure.

Next, we investigate the propagation of small-amplitude elastic waves in a first-order hierarchical honeycomb with $\gamma_{1}=$ 0.5 under different levels of applied compressive loads using FE simulations. The numerical analyses include (i) the stability analysis of the structure [36], (ii) the nonlinear postbuckling analysis of the system [36], and (iii) the propagation of small-amplitude elastic waves at a given deformation [22,37]. The 2D FE models were constructed using beam elements (hybrid element type B22H in ABAQUS). We assumed that the 2D phononic crystal is made of a silicon-based rubber (Elite Double 32:Zhermack) represented by an incompressible NeoHookean model [38], with $G_{s}=0.27 \mathrm{MPa}, K_{s}=13.4 \mathrm{MPa}$, and $\rho_{s}=965 \mathrm{~kg} / \mathrm{m}^{3}$ [39]. We chose this material to guarantee reversibility under large deformations.

Using this model, we calculate the dispersion relations for both undeformed and deformed configurations [24,29,37,40]. We report the normalized frequency $(\Omega)$ as a function of applied engineering strain $(\varepsilon)$ in Fig. 4(a) for uniaxial compression in the $y$ direction, Fig. 4(b) for uniaxial compression in the $x$ direction, and Fig. 4(c) for equibiaxial compression. Note that the structure was compressed in all of the directions up to the limit where the beams begin to contact one another. The dynamic response of the structure is characterized with four band gaps at the undeformed configuration in the given range of frequency $(0 \leqslant \Omega \leqslant 15)$. While the widest band gap at $\Omega=5.77-10.22$ remains almost unchanged during the entire range of applied strain, the other three band gaps are significantly altered upon compression in all of the directions. The lowest frequency band gap narrows and shifts to higher frequencies, whereas the other two high-frequency band gaps completely close around the buckling point and at $\varepsilon=-0.125$, respectively, for uniaxial and equibiaxial compressive loads. Furthermore, once buckling occurs (highlighted with dashed vertical lines), by increasing the level of deformation, several new band gaps open up at different levels of compression and most of them remain open, up to the highest level of applied deformation. The results reported in Fig. 4(c) clearly indicate that equibiaxial compression opens more band gaps, a signature of higher band-gap tunability (compared to other loading directions).

Moreover, the corresponding deformation mode shapes of the representative volume element (RVE) at different levels of applied strains were shown in Fig. 4. The results clearly show the emergence of distinct pattern transformations upon loading in different directions, induced by buckling of the individual beams. Since the pattern transformation is reversible, repeatable, and scale independent, our results provide insights into designing tunable materials and devices over a wide range of length scales.

In summary, our computational study, which investigates the influence of structural hierarchy and imposed deformations on band structure of self-similar hierarchical honeycombs, provides insights on the critical role of hierarchy on the dynamic response of phononic crystals. We found that hierarchy tends to shift the existing band gaps to lower frequencies while opening up new band gaps. Deformation was also demonstrated as another mechanism for opening more band gaps in hierarchical structures. This study, therefore, provides useful guidelines for the design of phononic devices with tunable properties [41-43].

This report was made possible by a NPRP award (NPRP 5-1298-2-560) from the Qatar National Research Fund (a member of the Qatar Foundation). 
[1] M. J. Buehler, Proc. Natl. Acad Sci. 103, 12285 (2006).

[2] J. L. Katz, A. Misra, P. Spencer, Y. Wang, S. Bumrerraj, T. Nomura, S. J. Eppell, and M. Tabib-Azar, Mater. Sci. Eng., C 27, 450 (2007).

[3] P. Fratzl and R. Weinkamer, Prog. Mater. Sci. 52, 1263 (2007).

[4] H. Qing and L. Mishnaevsky, Jr., Mech. Mater. 41, 1034 (2009).

[5] C. Ortiz and M. C. Boyce, Science 319, 1053 (2008).

[6] H. Yao and H. Gao, J. Mech. Phys. Solids 54, 1120 (2006).

[7] D. Ren, Y. Ma, Z. Li, Y. Gao, and Q. Feng, J. Cryst. Growth 325, 46 (2011).

[8] J. Aizenberg, J. C. Weaver, M. S. Thanawala, V. C. Sundar, D. E. Morse, and P. Fratzl, Science 309, 275 (2005).

[9] Y. Su, B. Ji, K. Zhang, H. Gao, Y. Huang, and K. Hwang, Langmuir 26, 4984 (2010).

[10] R. Oftadeh, B. Haghpanah, D. Vella, A. Boudaoud, and A. Vaziri, Phys. Rev. Lett. 113, 104301 (2014).

[11] R. Lakes, Nature (London) 361, 511 (1993).

[12] D. Rayneau-Kirkhope, Y. Mao, and R. Farr, Phys. Rev. Lett. 109, 204301 (2012)

[13] B. Haghpanah, J. Papadopoulos, D. Mousanezhad, H. NayebHashemi, and A. Vaziri, Proc. R. Soc. A 470, 20130856 (2014).

[14] D. Mousanezhad, H. Ebrahimi, B. Haghpanah, R. Ghosh, A. Ajdari, A.M.S. Hamouda, and A. Vaziri, Int. J. Solids Struct. 66, 218 (2015).

[15] S. Kinoshita, Structural Colors in the Realm of Nature (World Scientific, Singapore, 2008).

[16] L. P. Biro and J.-P. Vigneron, Laser Photon. Rev. 5, 27 (2011).

[17] A. D. Pris, Y. Utturkar, C. Surman, W. G. Morris, A. Vert, S. Zalyubovskiy, T. Deng, H. T. Ghiradella, and R. A. Potyrailo, Nat. Photon. 6, 195 (2012).

[18] J. Teyssier, S. V. Saenko, D. van der Marel, and M. C. Milinkovitch, Nat. Commun. 6, 6368 (2015).

[19] L. F. Deravi et al., J. R. Soc. Interface 11, 20130942 (2014).

[20] K. Bertoldi and M. C. Boyce, Phys. Rev. B 78, 184107 (2008).

[21] K. Jim, C. Leung, S. Lau, S. Choy, and H. Chan, Appl. Phys. Lett. 94, 193501 (2009).

[22] P. Wang, J. Shim, and K. Bertoldi, Phys. Rev. B 88, 014304 (2013).

[23] J. Shim, P. Wang, and K. Bertoldi, Int. J. Solids Struct. 58, 52 (2015).
[24] S. Gonella and M. Ruzzene, J. Sound Vib. 312, 125 (2008).

[25] A. S. Phani, J. Woodhouse, and N. Fleck, J. Acoust. Soc. Am. 119, 1995 (2006).

[26] X. Liu, G. Hu, C. Sun, and G. Huang, J. Sound Vib. 330, 2536 (2011).

[27] Y. Xu, C. Chen, and X. Tian, J. Vib. Acoust. 136, 011011 (2014).

[28] L. Brillouin, Wave Propagation in Periodic Structures: Electric Filters and Crystal Lattices (Dover Publications Inc., New York, 2003).

[29] See Supplemental Material at http://link.aps.org/supplemental/ 10.1103/PhysRevB.92.104304 for the information on undeformed and deformed configurations.

[30] D. Logan, A First Course in the Finite Element Method (Cengage Learning, Independence, KY, 2011).

[31] M. Maldovan and E. L. Thomas, Periodic Materials and Interference Lithography: For Photonics, Phononics and Mechanics (Wiley, New York, 2009).

[32] F. Casadei and J. Rimoli, Int. J. Solids Struct. 50, 1402 (2013).

[33] E. Nolde, R. Craster, and J. Kaplunov, J. Mech. Phys. Solids 59 , $651(2011)$

[34] S. Gonella and M. Ruzzene, Int. J. Solids Struct. 45, 2897 (2008).

[35] M. Maldovan and E. Thomas, Appl. Phys. B 83, 595 (2006).

[36] K. Bertoldi, M. Boyce, S. Deschanel, S. Prange, and T. Mullin, J. Mech. Phys. Solids 56, 2642 (2008).

[37] S. Babaee, P. Wang, and K. Bertoldi, J. Appl. Phys. 117, 244903 (2015).

[38] L. Treloar, Trans. Faraday Soc. 40, 59 (1944).

[39] S. Babaee, J. Shim, J. C. Weaver, E. R. Chen, N. Patel, and K. Bertoldi, Adv. Mater. 25, 5044 (2013).

[40] K. Bertoldi and M. Boyce, Phys. Rev. B 77, 052105 (2008).

[41] W. Cheng, J. Wang, U. Jonas, G. Fytas, and N. Stefanou, Nat. Mater. 5, 830 (2006).

[42] J. Vasseur, A.-C. Hladky-Hennion, B. Djafari-Rouhani, F. Duval, B. Dubus, Y. Pennec, and P. Deymier, J. Appl. Phys. 101, 114904 (2007).

[43] M. Maldovan, Nature (London) 503, 209 (2013). 


\title{
Supplementary Materials for
}

\author{
Honeycomb phononic crystals with self-similar hierarchy \\ Davood Mousanezhad ${ }^{1}$, Sahab Babaee ${ }^{2}$, Ranajay Ghosh ${ }^{1}$, \\ Elsadig Mahdi ${ }^{3}$, Katia Bertoldi ${ }^{2}$, and Ashkan Vaziri ${ }^{1, *}$ \\ ${ }^{1}$ Department of Mechanical and Industrial Engineering, Northeastern University, Boston, Massachusetts 02115, USA \\ ${ }^{2}$ School of Engineering and Applied Sciences, Harvard University, Cambridge, Massachusetts 02138, USA \\ ${ }^{3}$ Mechanical and Industrial Engineering Department, Qatar University, Doha, Qatar
}

\section{General formulations for undeformed configurations}

Here, we present fundamental relations and classical theories which are employed in the manuscript.

\subsection{Bloch wave theory}

In our study, we consider the structure to be infinitely extended in two-dimensional (2D) space. Fig. S1(a) shows the schematic of the primitive unit cell and the lattice vectors $\mathbf{a}_{1}$ and $\mathbf{a}_{2}$ for a second order hierarchical honeycomb. Note that the lattice vectors are the same for all orders of hierarchy and can be expressed as the following:

$$
\begin{gathered}
\mathbf{a}_{1}=\sqrt{3} \mathrm{l}_{0}(0.5 \mathbf{i}+0.5 \sqrt{3} \mathbf{j}) \\
\mathbf{a}_{2}=\sqrt{3} \mathrm{l}_{0}(-0.5 \mathbf{i}+0.5 \sqrt{3} \mathbf{j})
\end{gathered}
$$

where $\mathbf{i}$ and $\mathbf{j}$ are the Cartesian unit vectors in the $\mathrm{x}-\mathrm{y}$ plane. Note that all the vector and matrix quantities in this section are denoted by bold letters. The reciprocal lattice vectors (see Fig. S1(b)) are determined as the following: 
(a)

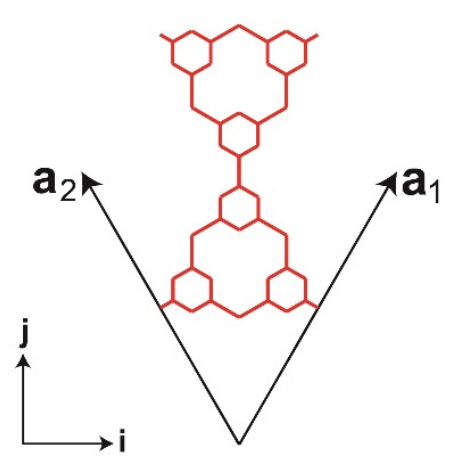

(b)

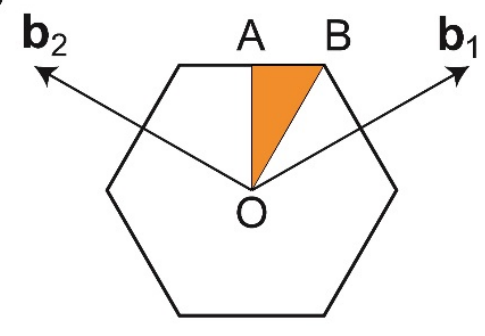

(c)

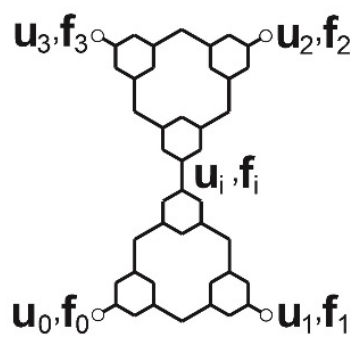

FIG. S1. (a) Schematic of the primitive unit cell and the lattice vectors $\left(\mathbf{a}_{\mathbf{1}}\right.$ and $\left.\mathbf{a}_{\mathbf{2}}\right)$ of a second order hierarchical honeycomb. (b) The reciprocal lattice vectors $\left(\mathbf{b}_{\mathbf{1}}\right.$ and $\left.\mathbf{b}_{\mathbf{2}}\right)$, first Brillouin zone (hexagonal area), and the irreducible Brillouin zone (shaded triangular area) of the structure. (c) Finite element model of the unit cell.

$$
\begin{aligned}
& \mathbf{b}_{1}=\frac{2 \pi}{3 \mathrm{l}_{0}}(\sqrt{3} \mathbf{i}+\mathbf{j}) \\
& \mathbf{b}_{2}=\frac{2 \pi}{3 \mathrm{l}_{0}}(-\sqrt{3} \mathbf{i}+\mathbf{j})
\end{aligned}
$$

Fig. S1(b) shows the first Brillouin zone (hexagonal region) corresponding to the hierarchical structure. It has been shown that the reflectional and rotational symmetries of the structure can further reduce the region to what referred as the irreducible Brillouin zone (IBZ) which for the hierarchical honeycomb is shown in Fig. $\mathrm{S} 1(\mathrm{~b})$ as a shaded triangular region (OAB). Furthermore, we can restrict the analysis to the wave vectors whose origins are located at the center of the first Brillouin zone $(\mathrm{O})$ with their tips located on the perimeter of the IBZ (O-A-BO). 


\subsection{Finite element formulations}

Numerical simulations of the propagation of small-amplitude elastic waves in the structures were performed using Finite Element (FE) method. Fig. S1(c) shows the unit cell of a second order hierarchical honeycomb structure used in FE analysis. We model the unit cell as a finite number of rigidly connected beam elements with axial, transverse, and rotational degrees of freedom (DOFs) whose mechanics is governed by Timoshenko beam theory [1]. A mesh sensitivity analysis was performed to ensure that the results are not dependent on the mesh size.

The FE form of unit cell's equation of motion can be expressed as:

$$
\left(\mathbf{K}-\omega^{2} \mathbf{M}\right) \mathbf{u}=\mathbf{f}
$$

where $\omega$ is the wave frequency, $\mathbf{K}$ and $\mathbf{M}$ are the global stiffness and mass matrices of the unit

cell, and, $\mathbf{u}$ and $\mathbf{f}$ are the vectors containing generalized nodal displacements and forces defined as the following:

$$
\begin{gathered}
\mathbf{u}=\left\{\begin{array}{lllll}
\mathbf{u}_{0} & \mathbf{u}_{1} & \mathbf{u}_{2} & \mathbf{u}_{3} & \mathbf{u}_{\mathrm{i}}
\end{array}\right\}^{\mathrm{T}} \\
\mathbf{f}=\left[\begin{array}{lllll}
\mathbf{f}_{0} & \mathbf{f}_{1} & \mathbf{f}_{2} & \mathbf{f}_{3} & \mathbf{f}_{\mathrm{i}}
\end{array}\right\}^{\mathrm{T}}
\end{gathered}
$$

where the subscripts $0,1,2$, and 3 denote the boundary nodes of the unit cell in contact with the adjacent cells, while the subscript i denote the internal nodes (see Fig. S1(c)). By virtue of Bloch theorem, the following periodic boundary conditions are obtained for the boundary nodes:

$$
\begin{aligned}
& \mathbf{u}_{2}=\mathrm{e}^{\mathrm{i} \mathrm{k}_{1}} \mathbf{u}_{0} \\
& \mathbf{u}_{3}=\mathrm{e}^{\mathrm{ik} k_{2}} \mathbf{u}_{1}
\end{aligned}
$$

and

$$
\mathbf{f}_{2}=-\mathrm{e}^{\mathrm{ik}} \mathbf{f}_{0}
$$




$$
\mathbf{f}_{3}=-\mathrm{e}^{\mathrm{ik}} \mathbf{f}_{1}
$$

Using Eq. 5, one can define the following transformation:

$$
\mathbf{u}=\mathbf{T} \mathbf{u}_{\mathrm{r}}
$$

where $\mathbf{u}_{\mathrm{r}}=\left\{\begin{array}{lll}\mathbf{u}_{0} & \mathbf{u}_{1} & \mathbf{u}_{\mathrm{i}}\end{array}\right\}^{\mathrm{T}}$ is the nodal displacements in the Bloch reduced coordinates, and $\mathbf{T}$ is a transformation matrix obtained as the following:

$$
\mathbf{T}=\left[\begin{array}{ccc}
\mathbf{I} & \mathbf{0} & \mathbf{0} \\
\mathbf{0} & \mathbf{I} & \mathbf{0} \\
\mathbf{I e}^{\mathrm{ik} k_{1}} & \mathbf{0} & \mathbf{0} \\
\mathbf{0} & \mathrm{Ie}^{\mathrm{ik}_{2}} & \mathbf{0} \\
\mathbf{0} & \mathbf{0} & \mathbf{I}
\end{array}\right]
$$

Now, substituting Eq. 7 into Eq. 3 and pre-multiplying the resulting equation by $\mathbf{T}^{\mathrm{H}}$, with ()$^{\mathrm{H}}$ denoting the Hermitian transpose, yields:

$$
\left[\mathbf{K}_{\mathrm{r}}\left(\mathrm{k}_{1}, \mathrm{k}_{2}\right)-\omega^{2} \mathbf{M}_{\mathrm{r}}\left(\mathrm{k}_{1}, \mathrm{k}_{2}\right)\right] \mathbf{u}_{\mathrm{r}}=\mathbf{0}
$$

where $\mathbf{K}_{\mathrm{r}}\left(\mathrm{k}_{1}, \mathrm{k}_{2}\right)$ and $\mathbf{M}_{\mathrm{r}}\left(\mathrm{k}_{1}, \mathrm{k}_{2}\right)$ denote the reduced stiffness and mass matrices. Eq. 9 is the equation of motion for free wave propagation which constitutes an eigenvalue problem whose solution expresses the dispersion characteristics of the periodic lattice. Wavenumbers $\mathrm{k}_{1}$ and $\mathrm{k}_{2}$, which for the wave propagation without attenuation are taken to be real quantities, vary within the first Brillouin zone. The solutions obtained for all possible values of $\mathrm{k}_{1}$ and $\mathrm{k}_{2}$ within the first Brillouin zone define the dispersion surfaces of the lattice. The number of dispersion surfaces corresponds to the number of reduced DOFs in the eigenvalue problem.

\subsection{Numerical implementations}

Matlab $^{\circledR}$ (Mathworks Inc., Natick, MA) was employed to develop a FE code to investigate the propagation of small-amplitude elastic waves in the proposed hierarchical honeycomb structure. Beams were assumed to have a rectangular cross section with unit length normal to the plane of 
wave motion and the thickness was adjusted to be consistent with the value of the relative density of the structure. Mesh convergence study was also performed in order to remove any mesh dependence on final results. Linear elastic properties of aluminum were assumed for the cell wall material with $E_{s}=71 \mathrm{GPa}, v_{\mathrm{s}}=0.33$, and $\rho_{\mathrm{s}}=2700 \mathrm{~kg} / \mathrm{m}^{3}$. In all the simulations in this study, the relative density is kept constant at $8 \%$.

We basically solved the eigenvalue problem given in Eq. 9 to obtain the dispersion relations $\omega=\omega(\mathbf{k})$ for $\mathbf{k}$ vectors varying on the perimeter of the IBZ. Then the band gaps were identified by the frequency ranges in which no solution exists for $\omega(\mathbf{k})$. At least fifty uniformly spaced points on each edge of the IBZ were used for band gap calculations. For convenience, the wave propagation frequency is normalized with respect to the first flexural frequency of a simply supported beam with length $l_{0}$ and thickness $t_{0}$, that is $\Omega=\omega / \omega_{0}$, where $\omega_{0}=\pi^{2} \sqrt{\mathrm{E}_{\mathrm{s}} \mathrm{t}_{0}^{2} /\left(12 \rho_{\mathrm{s}} \mathrm{l}_{0}^{4}\right)}[2]$.

\subsection{Additional results for low frequency directionality}

Note that the first and second modes of the propagating waves respectively represent shear- and pressure-dominated modes of propagation. The phase velocity $\mathbf{V}_{\mathrm{p}}$ and group velocity $\mathbf{V}_{\mathrm{g}}$ of the propagating waves can be calculated using the following relations:

$$
\begin{gathered}
\mathbf{V}_{\mathrm{p}}=\frac{\omega \mathbf{k}}{\|\mathbf{k}\|^{2}} \\
\mathbf{V}_{\mathrm{g}}=\frac{\partial \omega}{\partial \mathbf{k}}
\end{gathered}
$$

At least four hundred uniformly spaced points inside the IBZ were used to interpolate the dispersion surface in $\mathbf{k}$ space. 
Here, we first report phase and group velocity profiles for all directions of wave motion at $\Omega=0.1$ for the lowest two modes of propagating waves. Figs. S2, S3, S4, and S5 respectively present the phase and group velocity profiles for the first and second modes of propagating waves. The results are presented for six different values of $\eta$ (i.e. self-similarity ratio, defined in the manuscript) for self-similar hierarchical honeycombs up to third order of hierarchy. We employed the data extracted from these profiles to plot the evolution of anisotropy ratio against self-similarity ratio depicted in Fig. 3(d) in the manuscript. The results clearly indicate that the directionality of propagating waves at low frequency regimes is extremely dependent to the topology of the structure (i.e. the hierarchical order and self-similarity ratio). Then, the topology of the structure can be used as a design parameter to tune the directionality characteristics of the structure at low frequency regimes.

Next, we report in Figs. S6-S11 the contour plots of dispersion surfaces for the first (S-mode) and second (L-mode) modes of propagation. The results are presented for six different values of self-similarity ratio for self-similar hierarchical honeycombs up to third order of hierarchy. Note that $\xi_{1}$ and $\xi_{2}$ are the components of the wave vector along the Cartesian unit vectors in the $\mathrm{x}-\mathrm{y}$ plane, that is $\mathbf{k}=\mathrm{k}_{1} \mathbf{b}_{1}+\mathrm{k}_{2} \mathbf{b}_{2}=\xi_{1} \mathbf{i}+\xi_{2} \mathbf{j}$. The results again confirm the dependency of directionality of propagating waves at low frequency regimes to the topology of the structure. 

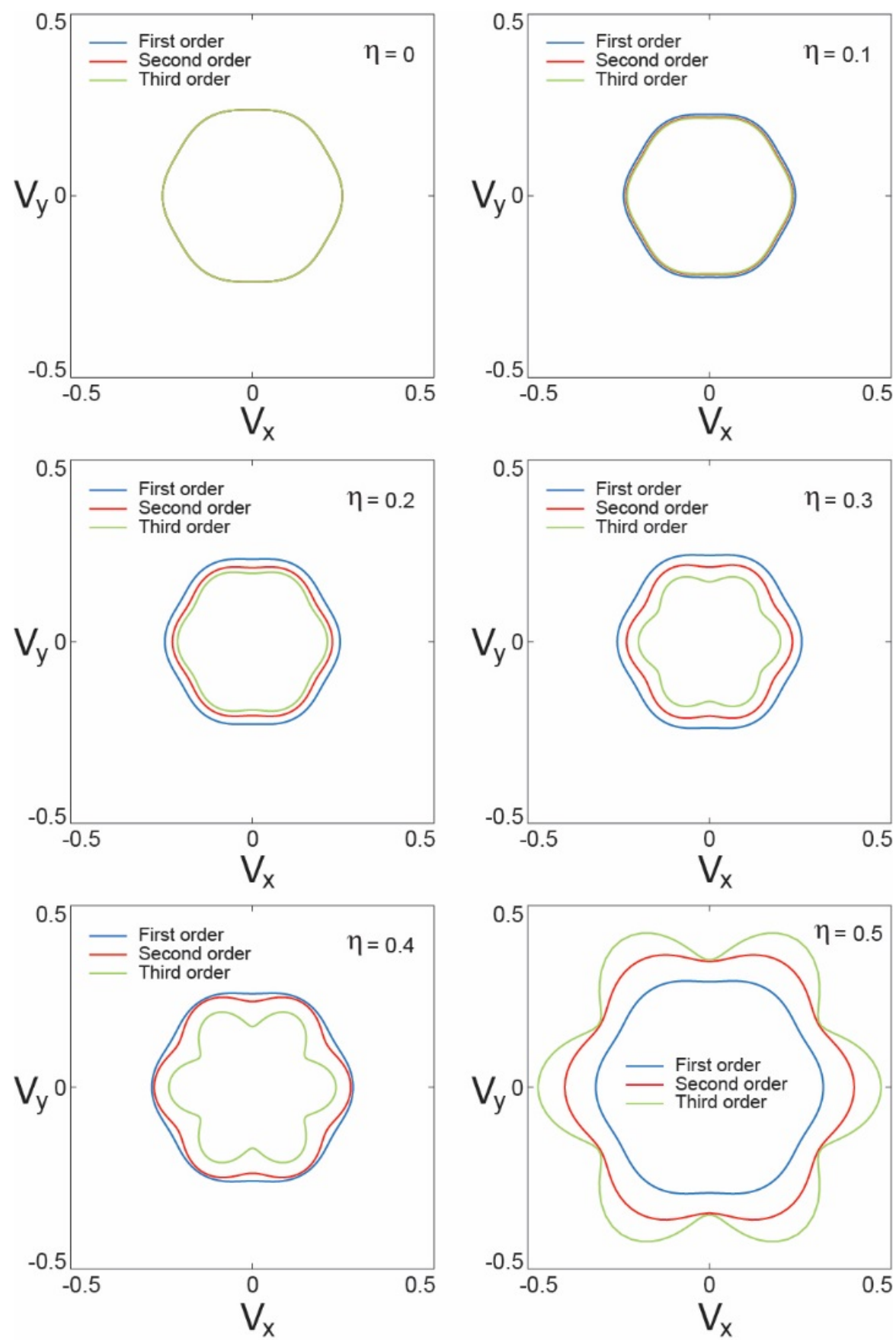

FIG. S2. Phase velocity profiles for the first mode of wave propagation. The results are presented for six different values of self-similarity ratio for self-similar hierarchical honeycombs up to third order of hierarchy. 

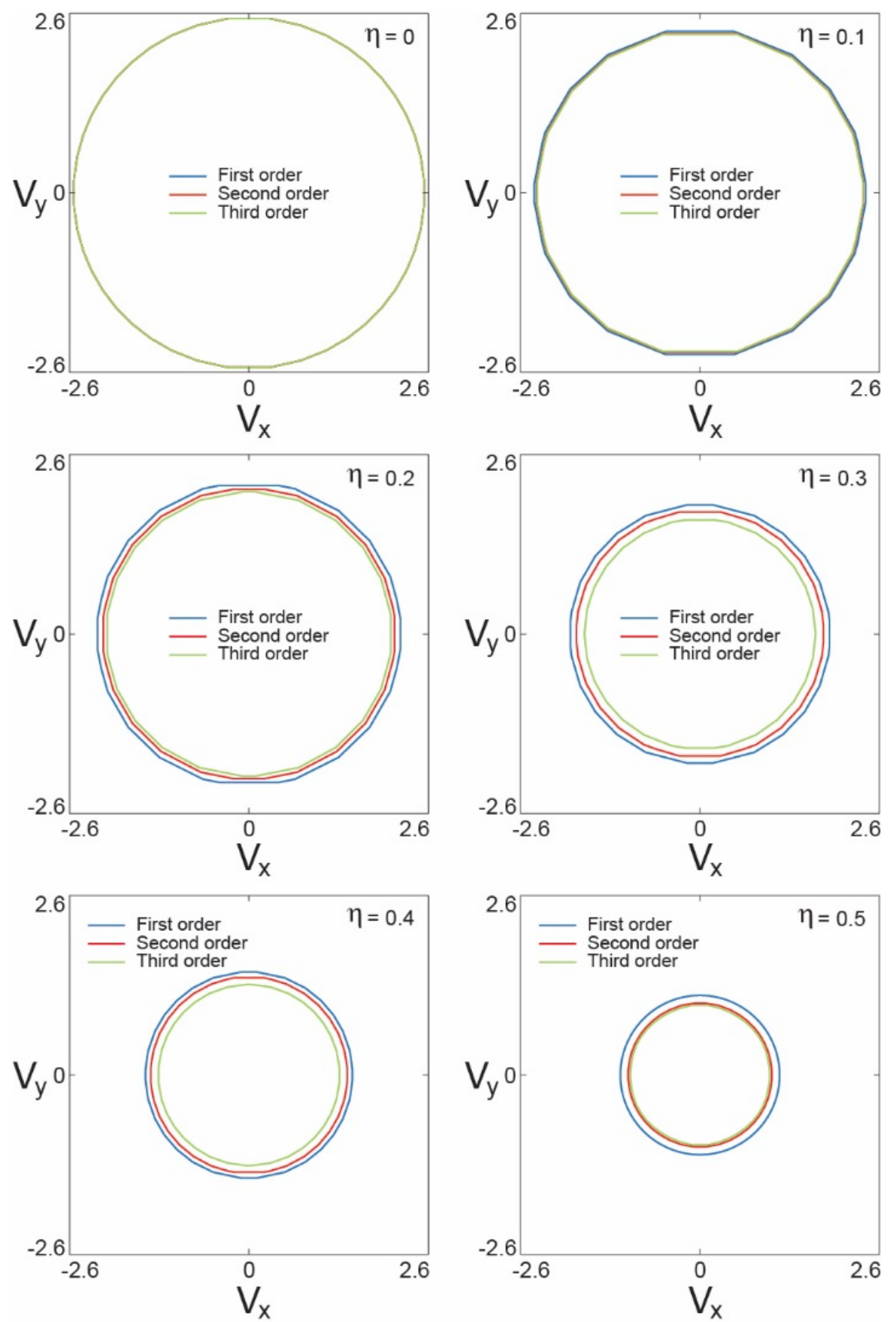

FIG. S3. Phase velocity profiles for the second mode of wave propagation. The results are presented for six different values of self-similarity ratio for self-similar hierarchical honeycombs up to third order of hierarchy. 

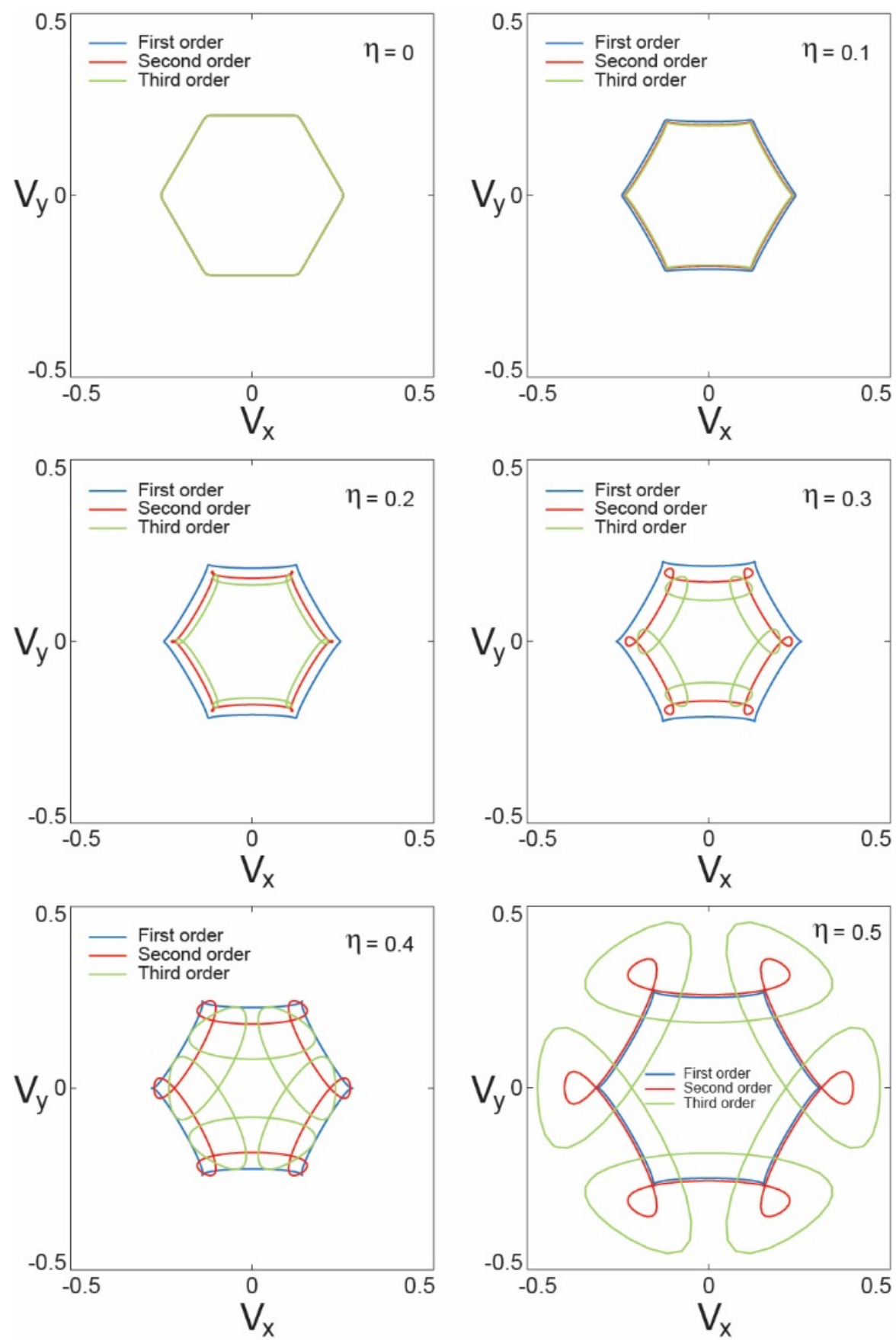

FIG. S4. Group velocity profiles for the first mode of wave propagation. The results are presented for six different values of self-similarity ratio for self-similar hierarchical honeycombs up to third order of hierarchy. 

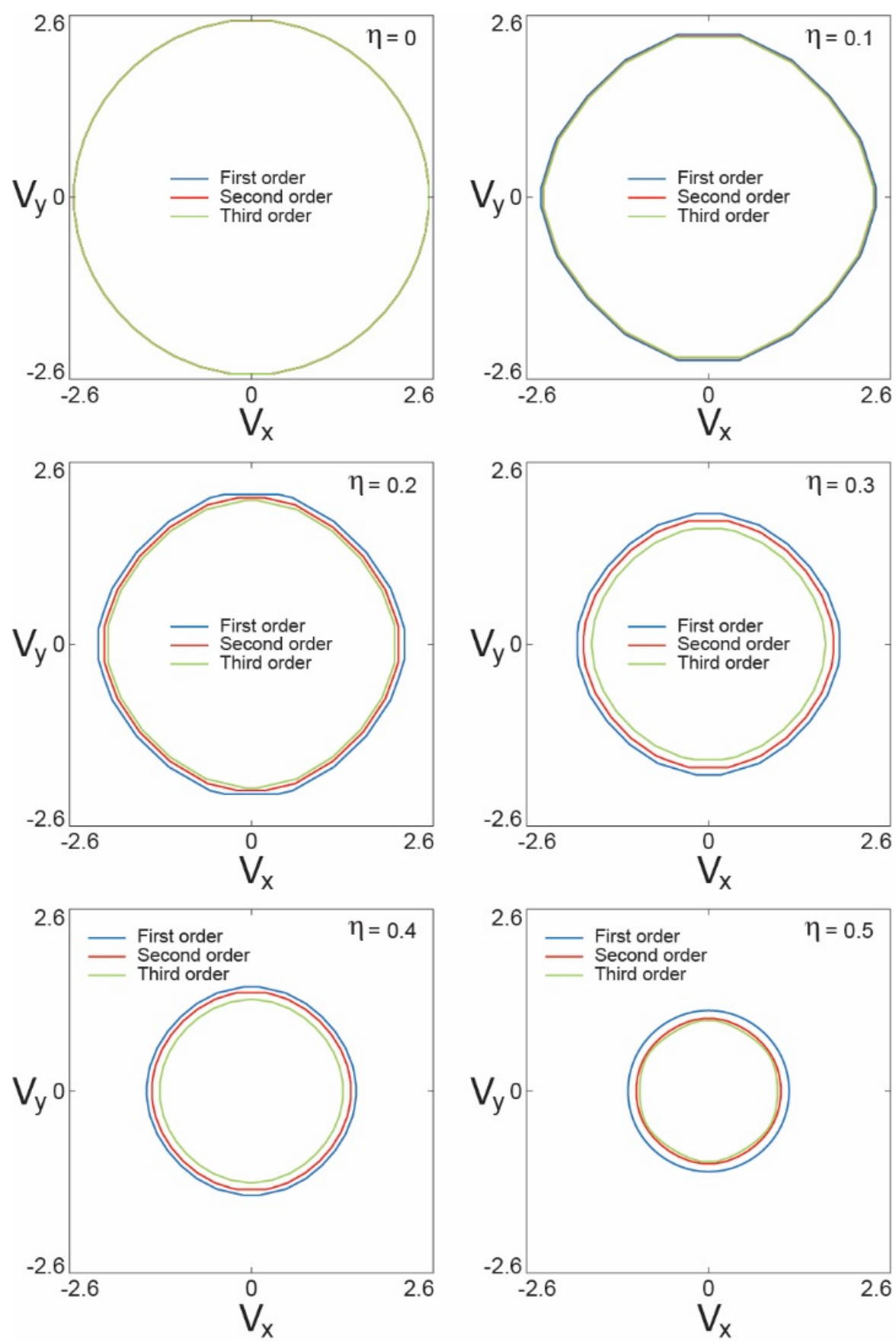

FIG. S5. Group velocity profiles for the second mode of wave propagation. The results are presented for six different values of self-similarity ratio for self-similar hierarchical honeycombs up to third order of hierarchy. 

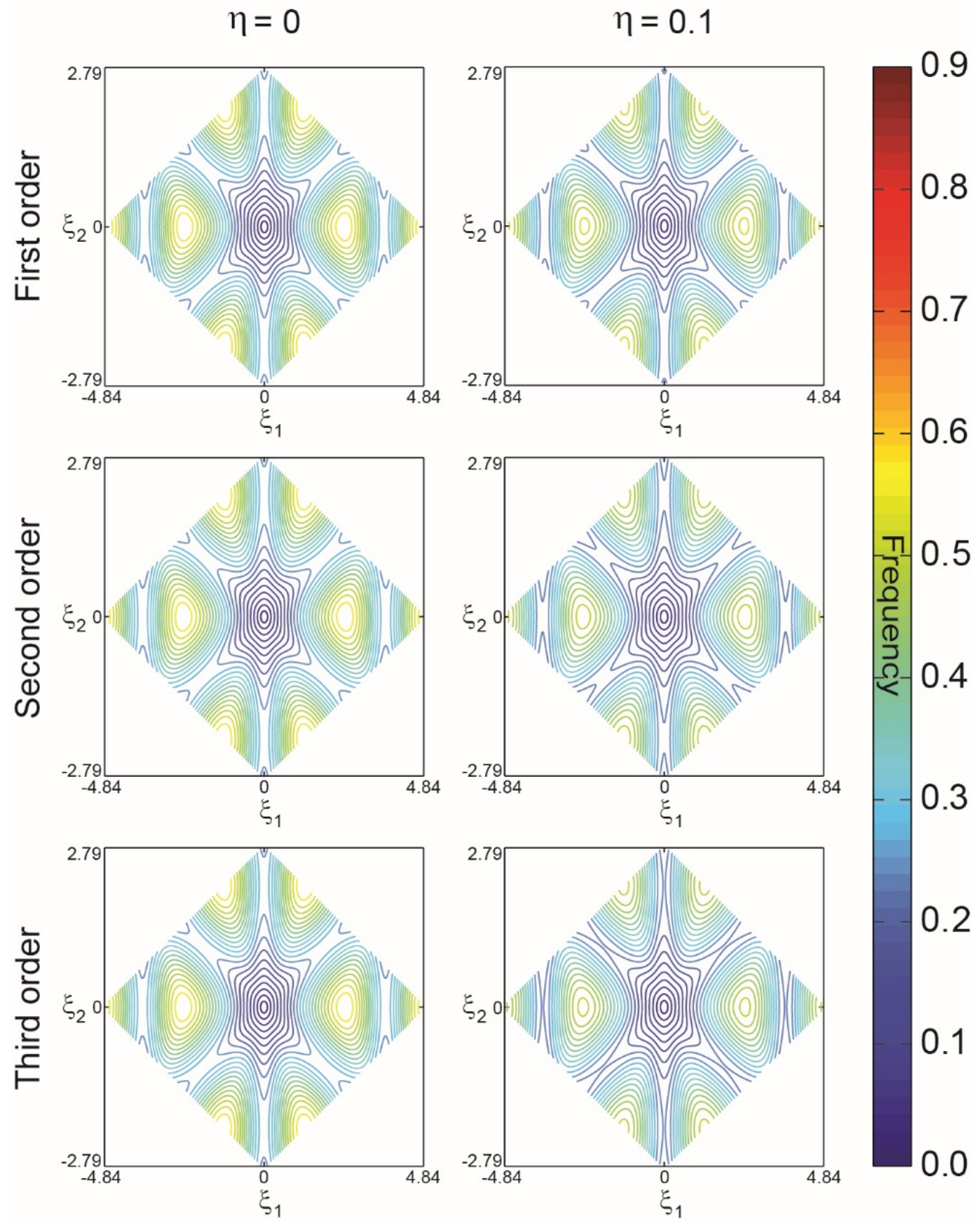

FIG. S6. Iso-frequency plots of the first mode of wave propagation. The results are presented for six different values of self-similarity ratio for self-similar hierarchical honeycombs up to third order of hierarchy. 

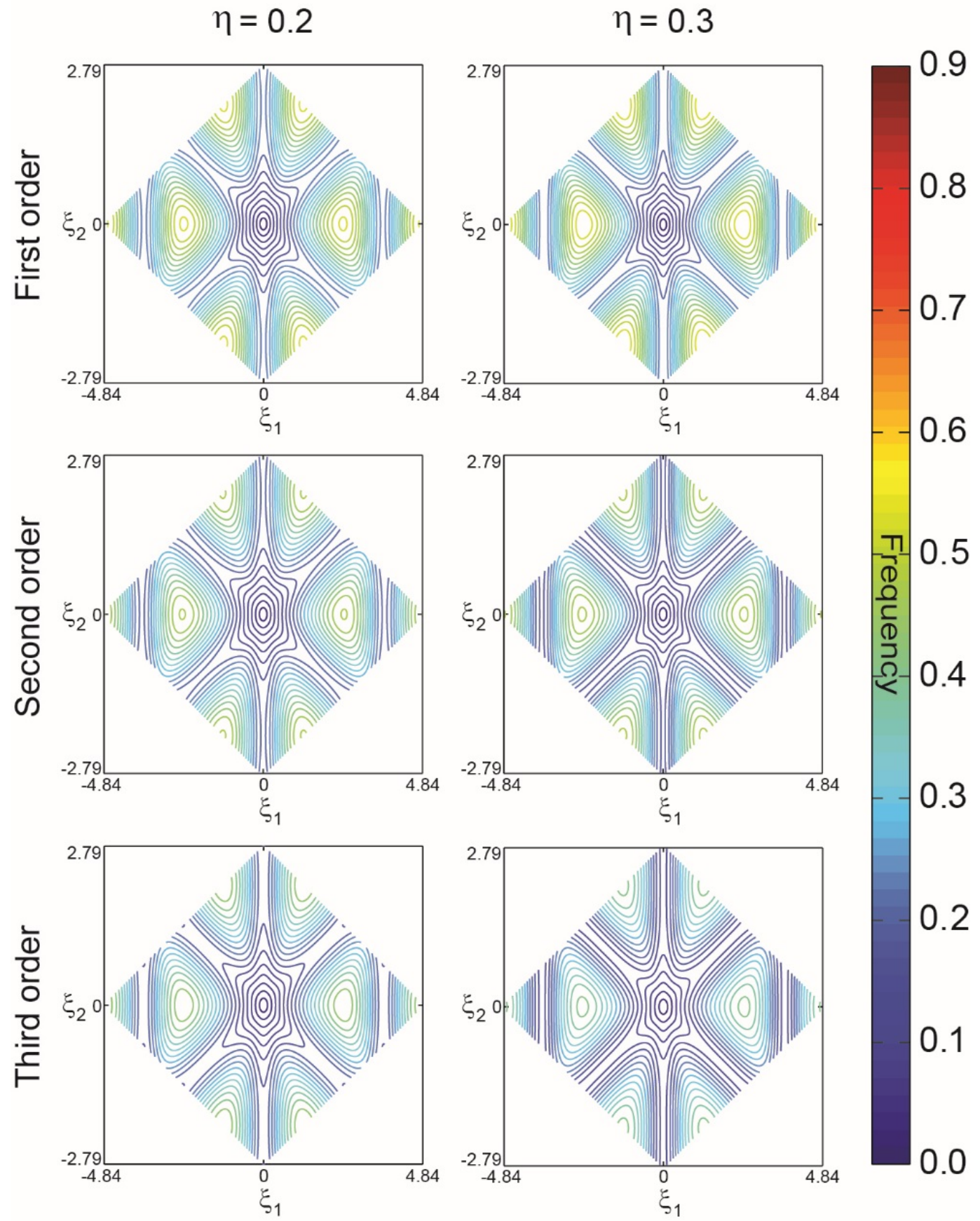

FIG. S7. Iso-frequency plots of the first mode of wave propagation. The results are presented for six different values of self-similarity ratio for self-similar hierarchical honeycombs up to third order of hierarchy. 

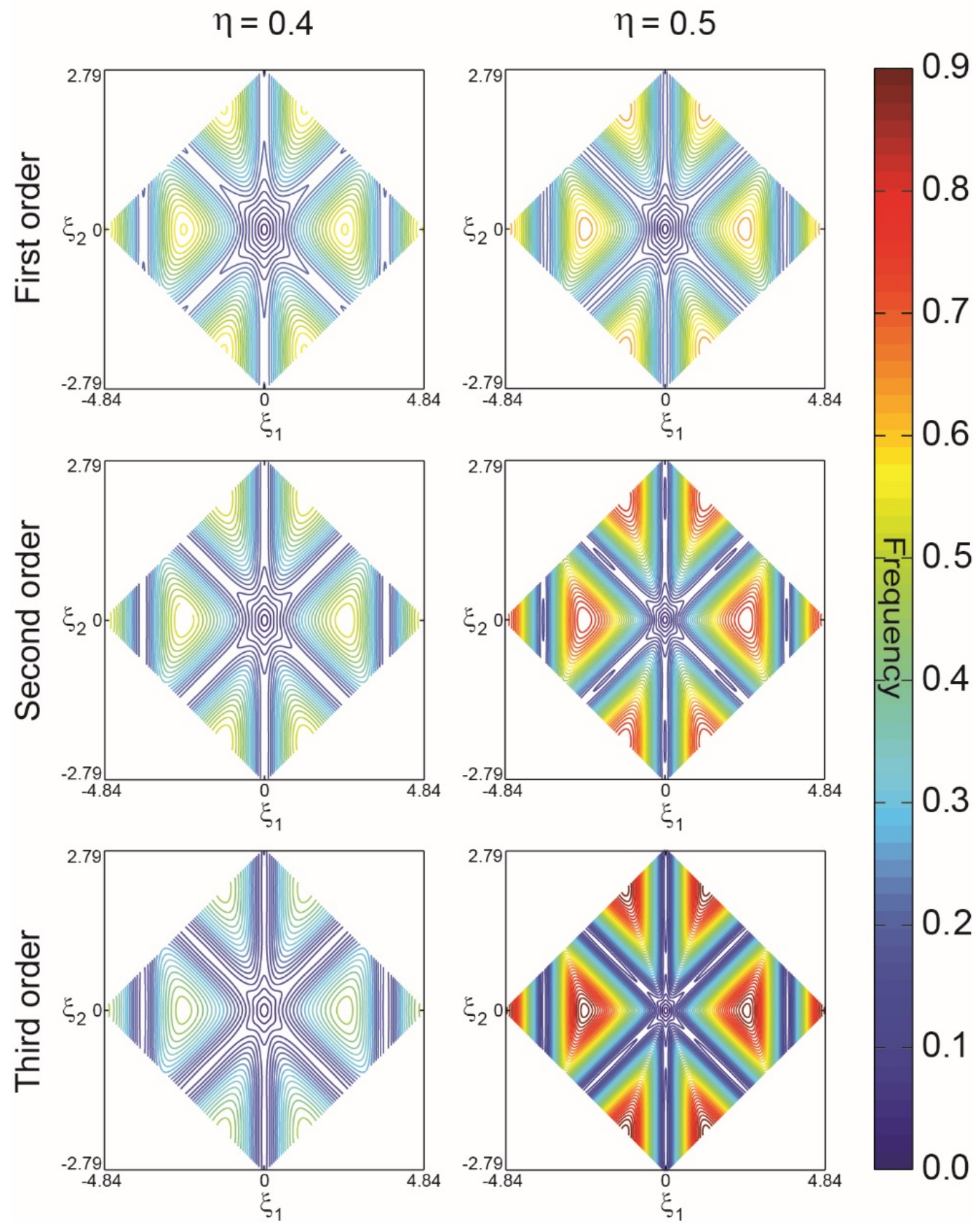

FIG. S8. Iso-frequency plots of the first mode of wave propagation. The results are presented for six different values of self-similarity ratio for self-similar hierarchical honeycombs up to third order of hierarchy. 

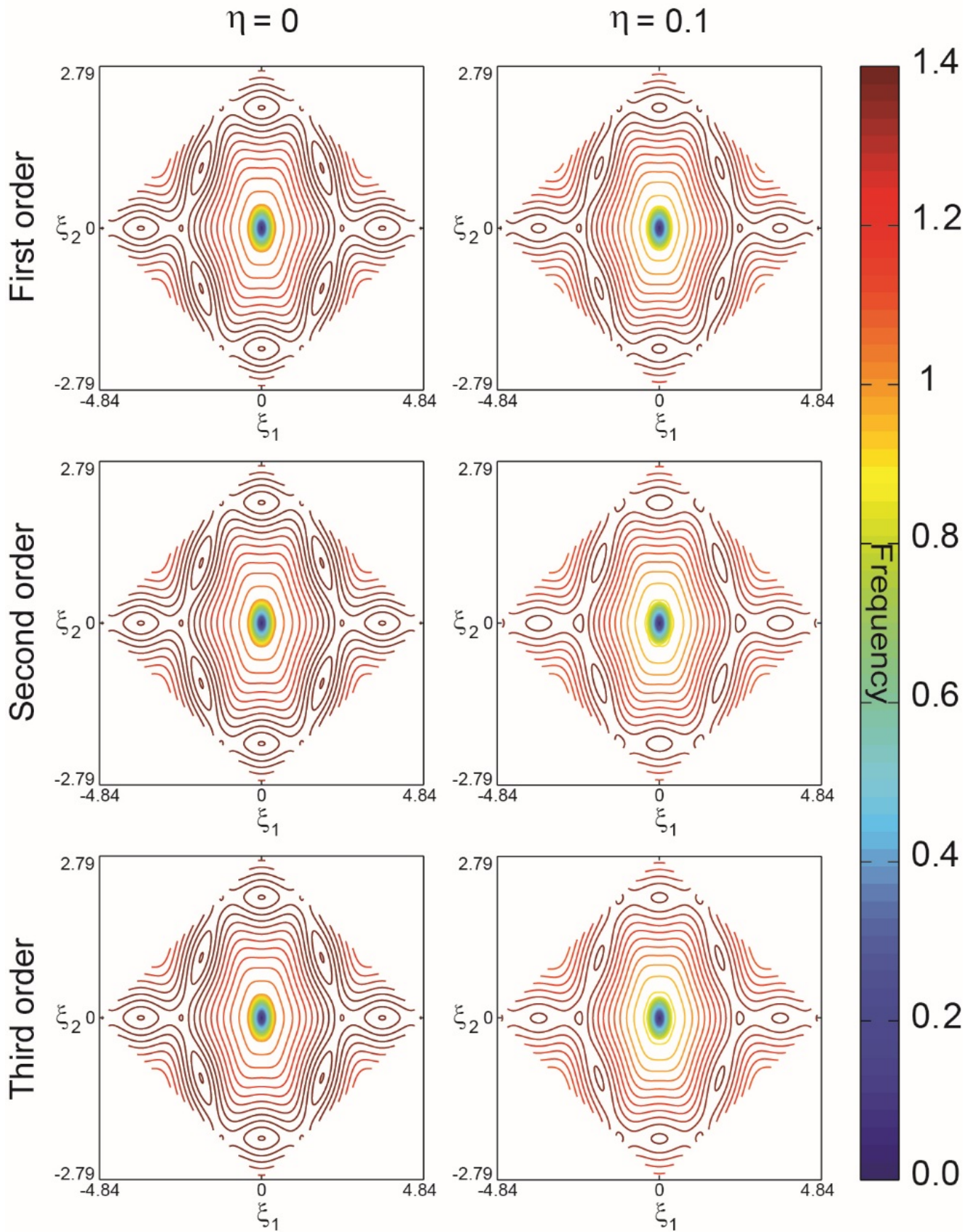

FIG. S9. Iso-frequency plots of the second mode of wave propagation. The results are presented for six different values of self-similarity ratio for self-similar hierarchical honeycombs up to third order of hierarchy. 

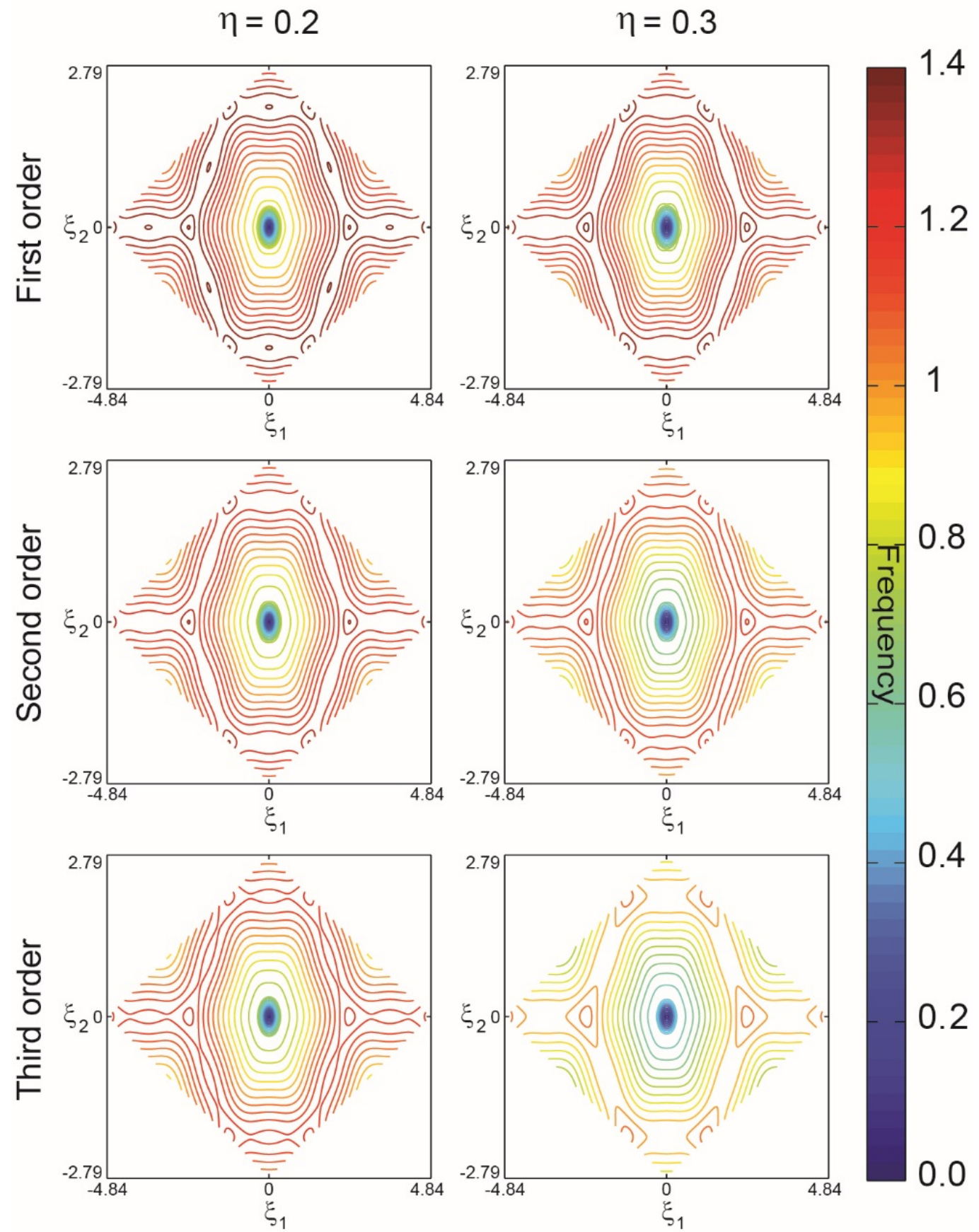

FIG. S10. Iso-frequency plots of the second mode of wave propagation. The results are presented for six different values of self-similarity ratio for self-similar hierarchical honeycombs up to third order of hierarchy. 

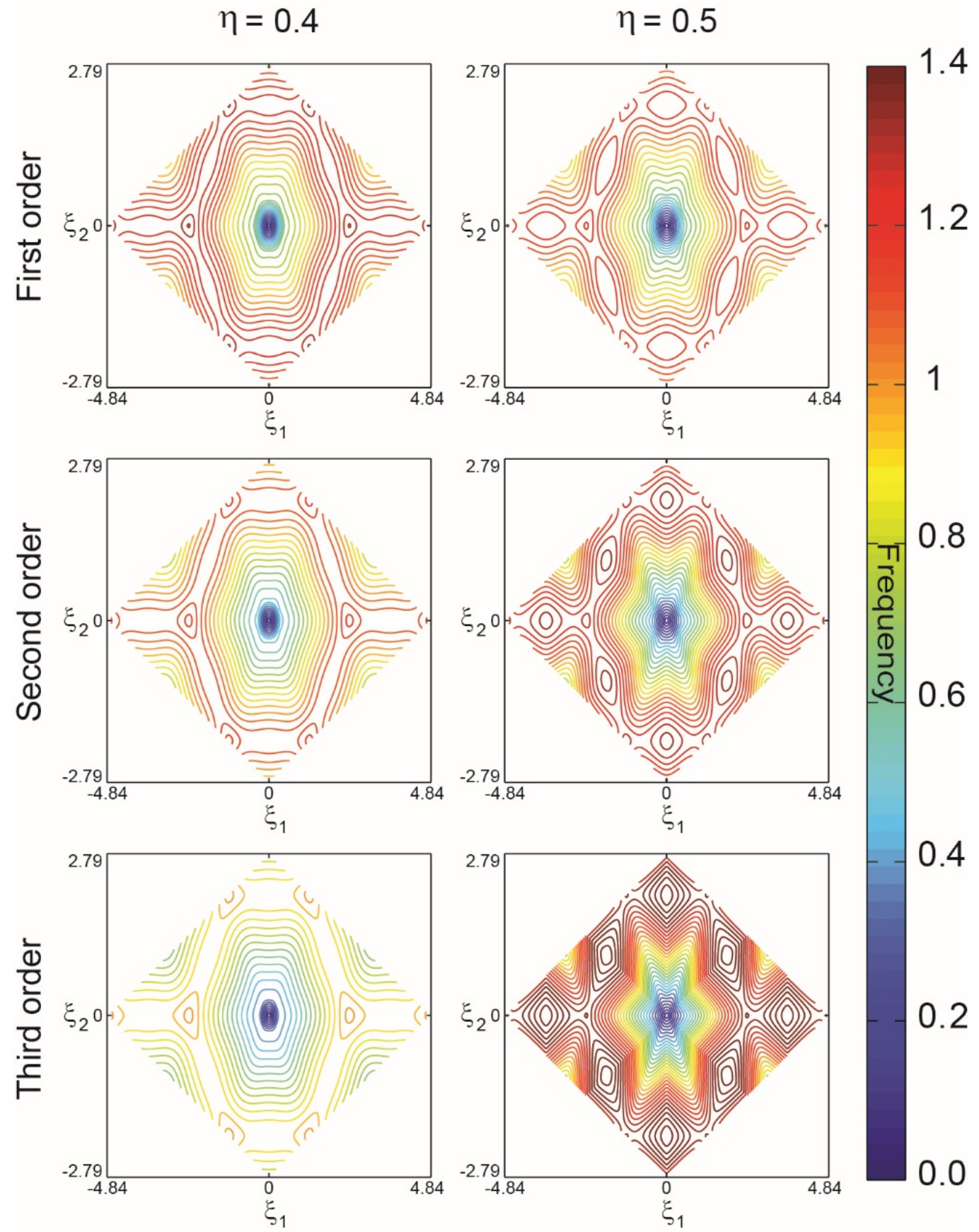

FIG. S11. Iso-frequency plots of the second mode of wave propagation. The results are presented for six different values of self-similarity ratio for self-similar hierarchical honeycombs up to third order of hierarchy. 


\section{Effect of deformation; wave propagation analysis}

We investigated the propagation of small-amplitude elastic waves in an elastomeric deformable hierarchical honeycomb with $\gamma_{1}=0.5$ under different levels of applied compression in $x$-, $y$-, and biaxial directions. In order to obtain the dispersion relation, $\omega=\omega(k)$, frequency domain wave propagation analysis was conducted on both the undeformed and deformed configurations generated by the post-buckling analysis using commercial FE package ABAQUS/Standard [3,4].

In this section, for the sake of simplicity in numerical calculations, we considered a rectangular unit cell, and we performed the simulations on the enlarged unit cell assuming Bloch type boundary conditions which is implemented using a user defined multiple point constraint (MPC) subroutine [3-5]. In the band diagrams presented in Figs. S13, S14, and S15, the normalized frequency $(\Omega)$ was calculated for all $k$ vectors along the perimeter and the diagonal of the IBZ in reciprocal lattice space (see path $G-X-M-G-Y-M$ in Fig. S12). We used 20 uniformly spaced $k$ points on each line segment (e.g. $\mathrm{G}-\mathrm{X}$ ), that they were updated at every levels of deformation [6]. 
(a)

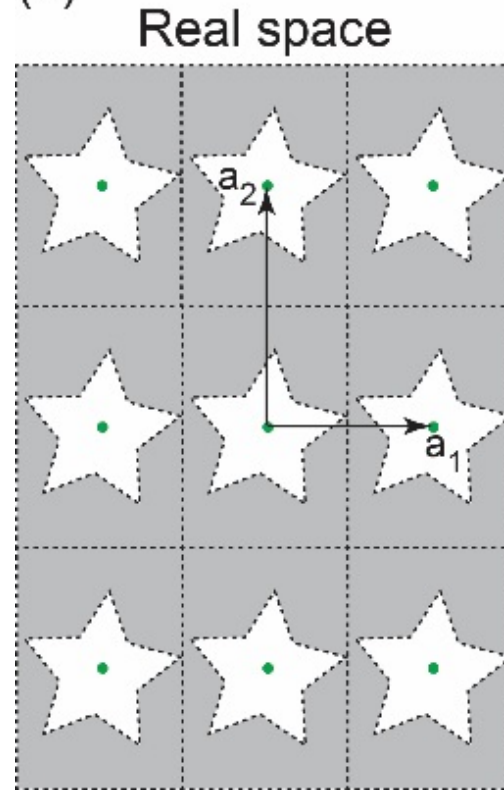

(b)

\section{Reciprocal space}

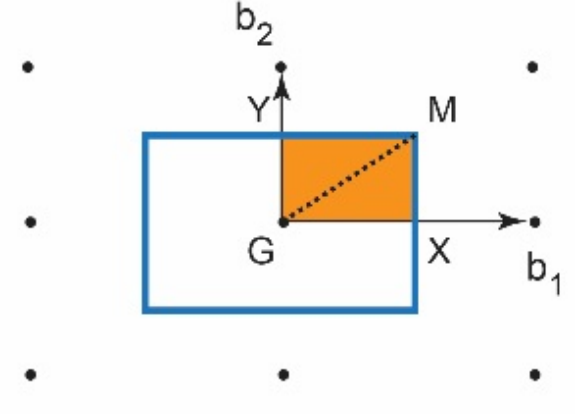

FIG. S12. Schematic of a 2D point lattice in (a) real space (green dots), and (b) reciprocal space (black dots). The unit cell spanned by lattice vectors $a_{1}$ and $a_{2}$ is shown in grey. The first Brillioun zone (blue rectangle area) and the irreducible Brillioun zone (orange area GXMYM) are shown in (b). 

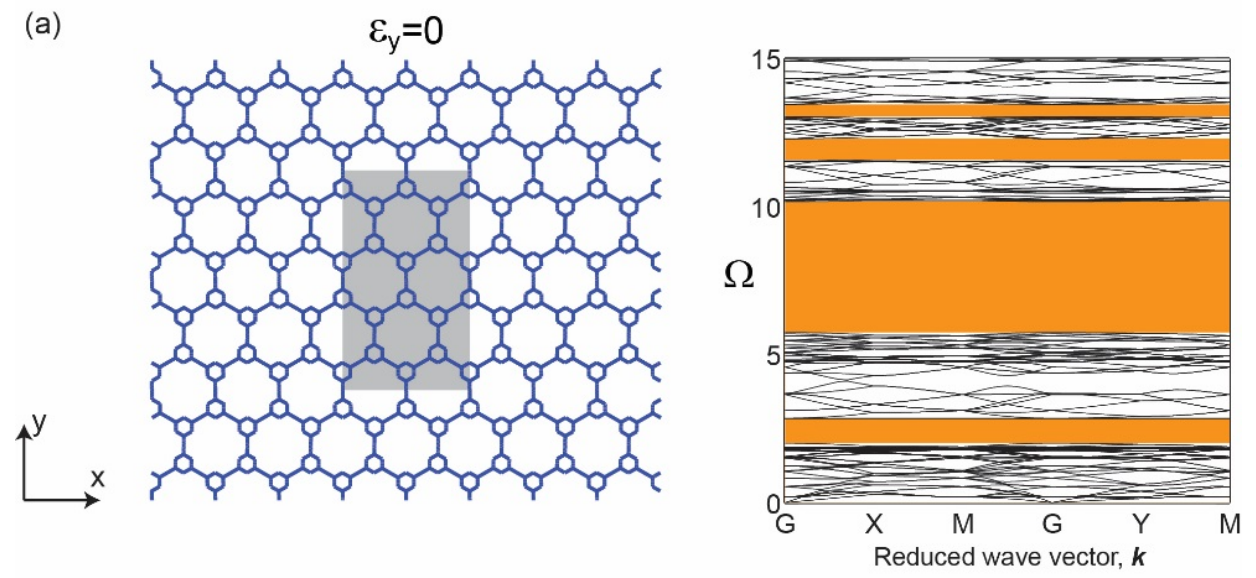

(b)
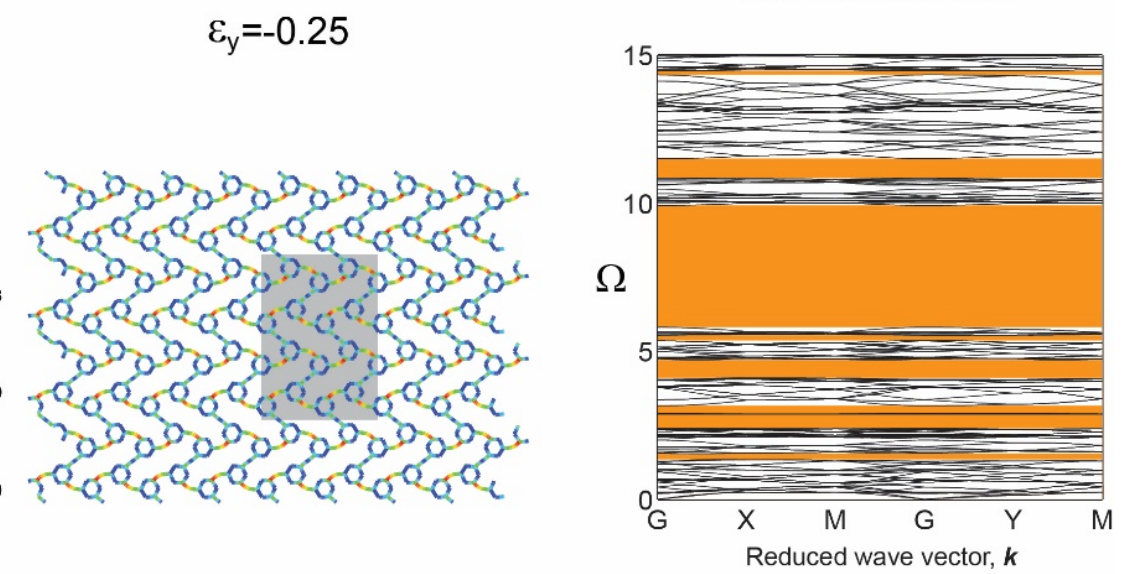

(c)

$$
\varepsilon_{\mathrm{y}}=-0.5
$$
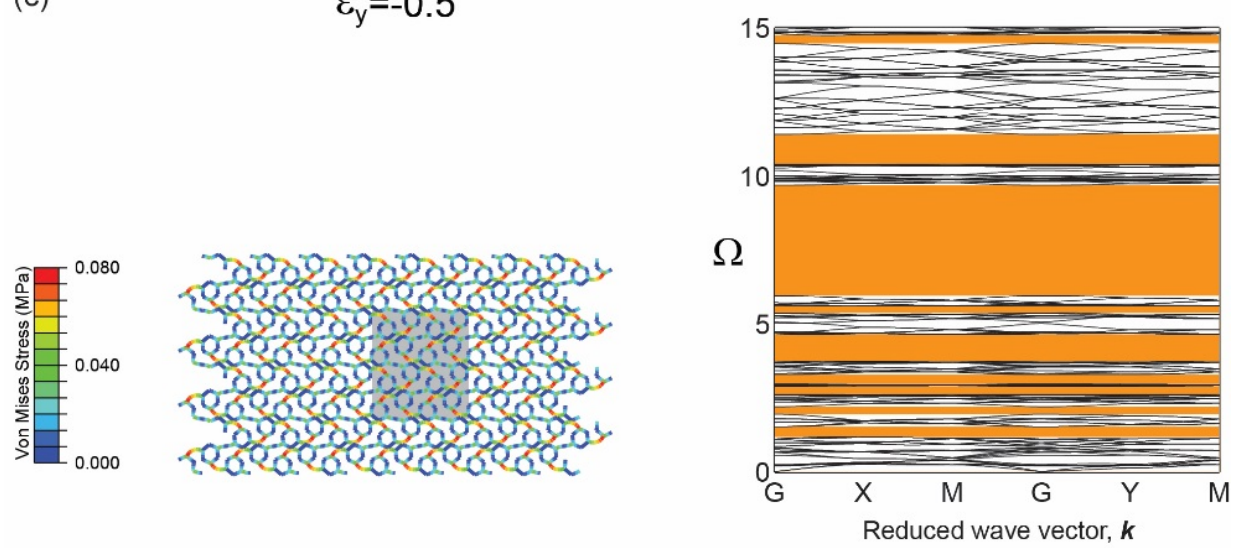

FIG. S13. Effect of the applied compression in y-direction on propagation of elastic waves. The band diagrams and corresponding deformed structures are presented at three different levels of applied deformation. The contour plot on the deformed structures represents the von Mises stress distribution (unit:MPa), and the dark grey area shows the RVE during the pattern transformation. 

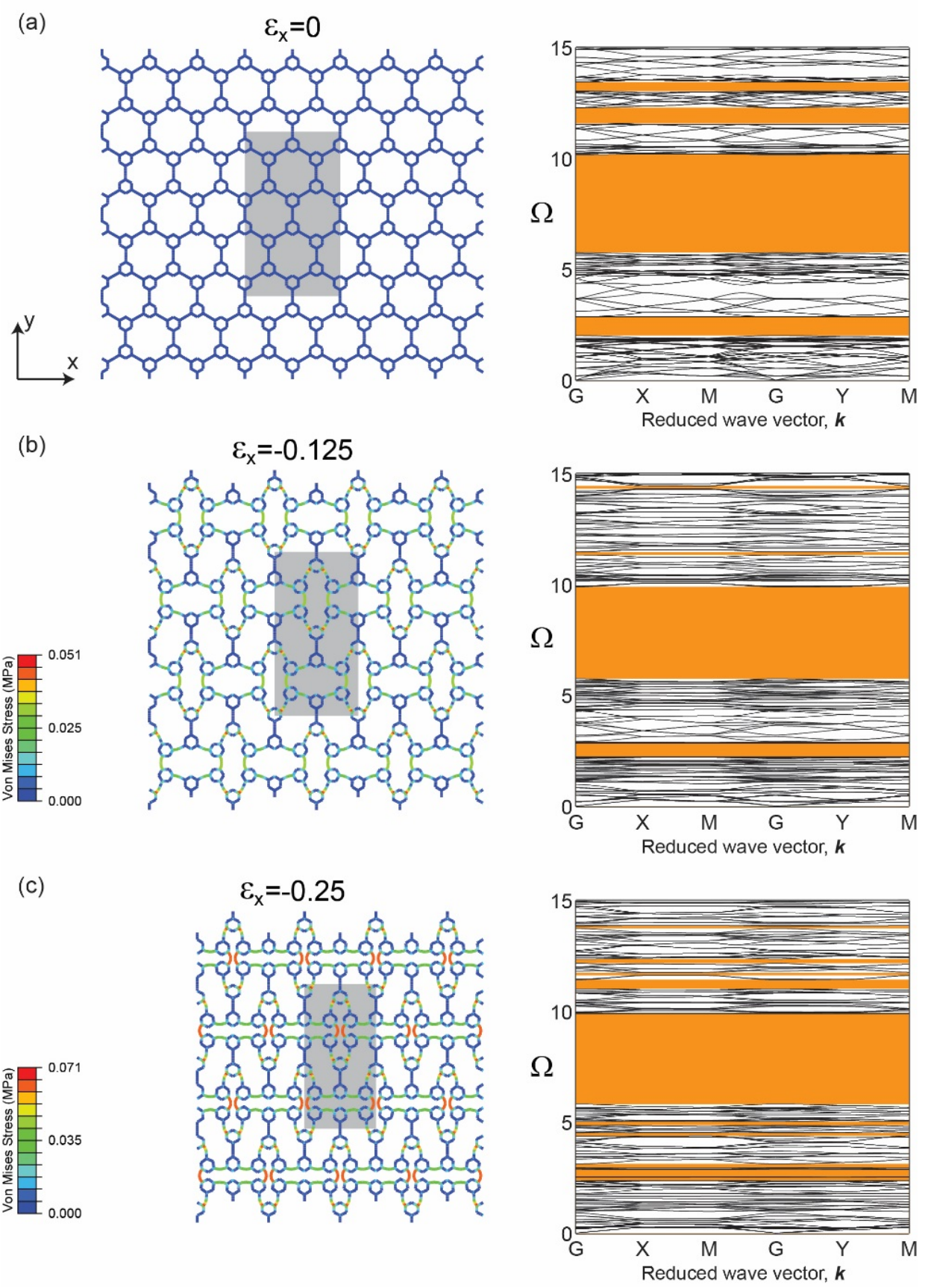

FIG. S14. Effect of the applied compression in x-direction on propagation of elastic waves. The band diagrams and corresponding deformed structures are presented at three different levels of applied deformation. The contour plot on the deformed structures represents the von Mises stress distribution (unit:MPa), and the dark grey area shows the RVE during the pattern transformation. 

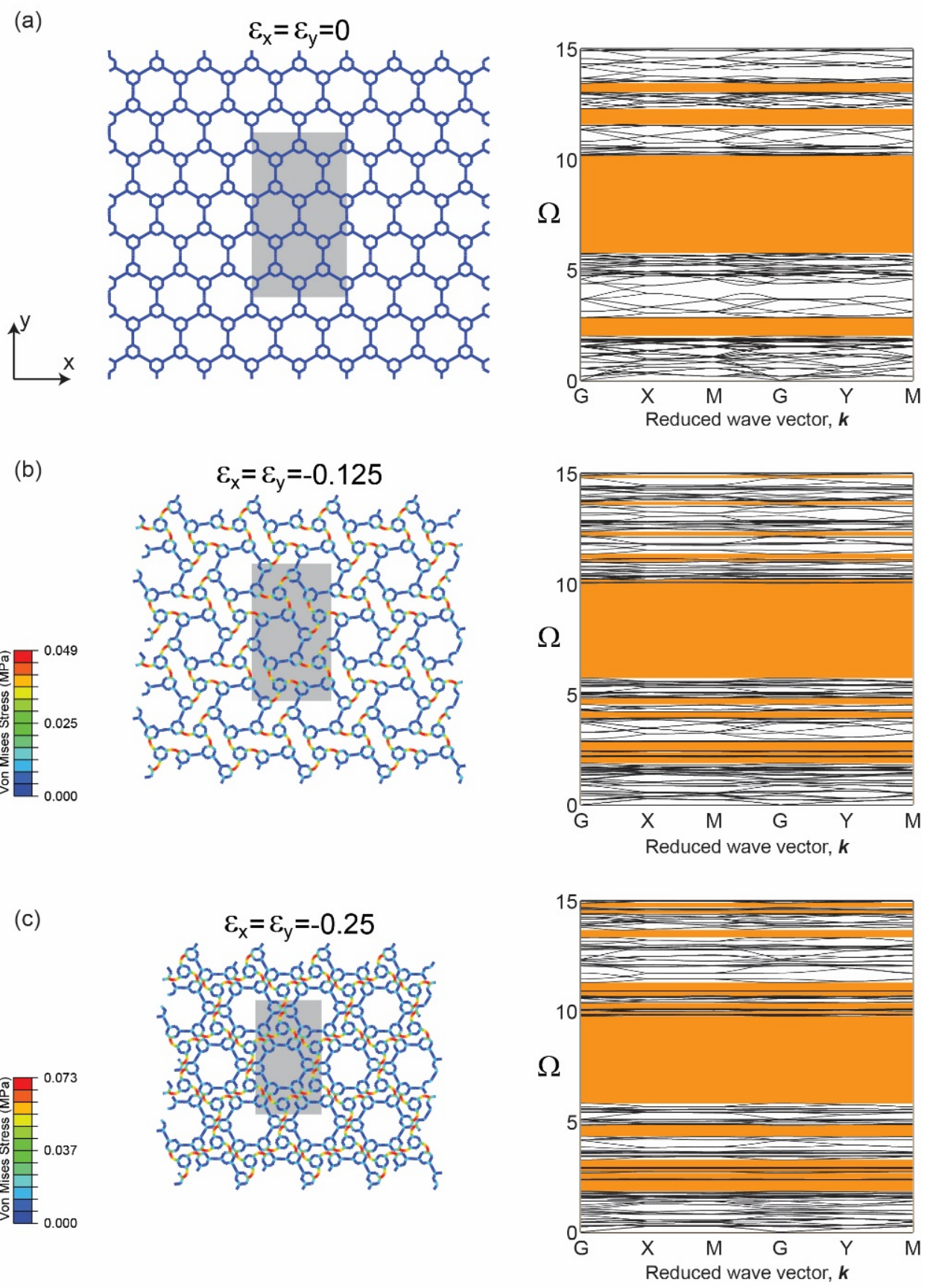

FIG. S15. Effect of the applied eqi-biaxial compression on propagation of elastic waves. The band diagrams and corresponding deformed structures are presented at three different levels of applied deformation. The contour plot on the deformed structures represents the von Mises stress distribution (unit:MPa), and the dark grey area shows the RVE during the pattern transformation. 
*vaziri@coe.neu.edu

Refrences

[1] D. Logan, A first course in the finite element method (Cengage Learning, 2011).

[2] A. S. Phani, J. Woodhouse, and N. Fleck, The Journal of the Acoustical Society of America 119, 1995 (2006).

[3] S. Babaee, P. Wang, and K. Bertoldi, Physical review B (2015).

[4] K. Bertoldi and M. Boyce, Physical Review B 77, 052105 (2008).

[5] P. Wang, J. Shim, and K. Bertoldi, Physical Review B 88, 014304 (2013).

[6] J. Shim, P. Wang, and K. Bertoldi, International Journal of Solids and Structures (2014). 